Open Access

\title{
Robust widely linear beamforming using estimation of extended covariance matrix and steering vector
}

\author{
Zhen Meng ${ }^{1}$, Weidong Zhou ${ }^{\text {* }}$ (1) and Saeed Gazor ${ }^{2}$
}

\author{
${ }^{*}$ Correspondence: \\ zhouweidong@hrbeu.edu.cn \\ ${ }^{1}$ College of Automation, Harbin \\ Engineering University, 150001 \\ Harbin, People's Republic of China \\ Full list of author information is \\ available at the end of the article
}

\begin{abstract}
The distribution of the received signals in many array processing applications is noncircular. Although optimal widely linear beamformer (WLB) can provide the best performance for noncircular received signals, its performance degrades severely under model mismatches in practical applications. As a remedy, we propose a robust WLB by using precise reconstruction of extended interference-plus-noise covariance matrix (EINCM) and low-complexity estimation of extended desired signal steering vector (EDSSV). We propose to first determine the steering vectors, powers, and noncircularity coefficients of all signals and the noise power. In contrast to the previous reconstruction methods using the integration over a wide angular sector, we reconstruct the interference-plus-noise covariance matrix (INCM) and the pseudo INCM accurately according to their definitions. By using INCM and pseudo INCM, we can precisely reconstruct the EINCM. We propose to estimate the EDSSV by intersecting two extended subspaces, which are respectively formed by eigendecomposing the extended sample covariance matrix and the extended desired signal covariance matrix. Unlike the convex optimization methods, the proposed EDSSV estimation does not require any optimization programming and yields a solution with closed expression in low computational complexity. Simulation results show that the proposed robust WLB provides near optimal performance under several model mismatch cases.
\end{abstract}

Keywords: Noncircular signal, Robust widely linear beamforming, Noncircularity coefficient, Extended covariance matrix, Subspace intersection, Extended steering vector

\section{Introduction}

Adaptive beamforming aims at extracting desired signal (DS) while suppressing interferences and noise and is a fundamental technique in array signal processing [1-5]. Traditional adaptive beamformers mostly focus on second-order circular signals with stationary observations, such as minimum variance distortionless response (MVDR) beamformer and linearly constrained minimum variance (LCMV) beamformer [6-10]. The second-order noncircular and nonstationary signals frequently appear in the fields of radio communication and satellite communication, such as unbalanced quaternary

(C) The Author(s). 2020 Open Access This article is licensed under a Creative Commons Attribution 4.0 International License which permits use, sharing, adaptation, distribution and reproduction in any medium or format, as long as you give appropriate credit to the original author(s) and the source, provide a link to the Creative Commons licence, and indicate if changes were made. The images or other third party material in this article are included in the article's Creative Commons licence, unless indicated otherwise in a credit line to the material. If material is not included in the article's Creative Commons licence and your intended use is not permitted by statutory regulation or exceeds the permitted use, you will need to obtain permission directly from the copyright holder. To view a copy of this licence, visit http://creativecommons.org/licenses/by/4.0/. 
phase shift keying (UQPSK), amplitude modulated (AM), amplitude-shift keying (ASK), and binary phase-shift keying (BPSK) signals [11-13]. However, these beamformers become suboptimal when they encounter noncircular signals. In such cases, the optimal beamforming shall be as widely linear (WL) [14-20].

A variety of widely linear beamformers (WLBs) have been developed in the past decade to exploit the noncircularity of noncircular signals. In [21], a WL MVDR beamformer is introduced which outperforms the traditional MVDR beamformer for noncircular interferences. However, the beamformer in [21] ignores the noncircularity of the DS. As a result, this beamformer cannot fully exploit the noncircularity information which becomes suboptimal. To fully utilize the noncircularity of DS, an optimal WL MVDR is proposed in [22] where more DS components are retrieved through the orthogonal decomposition of the conjugate DS. The optimal WL MVDR beamformer has better performance than the WL MVDR beamformer and its excellent performance is further analyzed in $[23,24]$. However, the optimal WL MVDR beamformer relies on the predefined noncircularity coefficient and the exact desired signal steering vector (DSSV), which are not available in practical applications. Many unideal factors will lead to the DSSV mismatches, such as imperfect array calibration, wavefront distortion, local scattering, and look direction error. The mismatch of noncircularity coefficient is often caused by phase offset, frequency offset, and partial waveform information of DS. The WL MVDR beamformer will suffer serious performance degradation due to these mismatches.

The robust WLBs have been proposed to improve the robustness against various mismatches. A robust method for the optimal WL MVDR beamformer is proposed in [25] to combat the mismatches of the noncircularity coefficient and the DSSV. However, this robust WLB is sensitive to the large mismatch of noncircularity coefficient. A noncircularity coefficient estimator is proposed in [26] by only using the noncircular DSSV. However, this robust WLB relies on the exact DSSV and it is not effective in DSSV mismatch case. The authors in [27] extend the robust Capon beamformer to the generalized case with noncircular DS and noncircular interferences. Although this WLB is robust against the errors in steering vector, sample covariance matrix (SCM), and DS noncircularity coefficient, its performance degrades greatly in high signal-to-noise ratios (SNRs). In [28], the authors propose two WL minimum dispersion beamformers by fully utilizing the noncircularity and sub-Gaussianity of signals to improve the beamforming performance. A class of DS noncircularity coefficient estimators for WLB are proposed in [29], which employ the algebraic structure of the extended covariance matrix in different viewpoints. These methods have excellent performance in different scenarios of low SNR and few numbers of snapshots. In [30], a spatial spectrum of noncircularity coefficient (SSNC) is estimated and the extended interference-plus-noise covariance matrix (EINCM) is reconstructed based on the Capon power and noncircularity coefficient spectra. This robust WLB has robustness against look direction error and steering vector mismatch. However, it requires the precise array sensor geometry. A robust WLB is proposed in [31] which prevents the extended desired signal steering vector (EDSSV) from converging to the interferences based on a projection constraint (PC). Three robust WLBs are proposed in [32] by reconstructing the EINCM via modifying the SSNC and by estimating the EDSSV using three different estimators. The EINCM outperforms the extended SCM because the EINCM reduces the DS self-nulling effects that are usually caused by the extended SCM. The robust WLBs using EINCM achieve near optimal WL beamforming 
performance while the WLBs using extended SCM suffer from performance degradation at high SNRs. However, the existing EINCM reconstruction is still not precise because the exploited Capon spatial spectrum is sensitive to array perturbation and the integration angular sector is too wide. Moreover, the computational complexity of the existing EDSSV estimation is very high because it requires convex optimization programming.

In this paper, we propose a precise method for the EINCM reconstruction and an inexpensive and accurate method for the EDSSV estimation. Together, these methods lead to a robust WLB with significant performance improvement which is computationally efficient. We propose to compute the steering vectors of all signals by extending the iterative robust Capon beamformer (IRCB) and propose to estimate the powers of all signals by employing the covariance fitting approach. In our proposed method, the noncircularity coefficients of all signals are estimated by extending the DS noncircularity coefficient estimator and the noise power is estimated as the minimum eigenvalue of SCM. We propose to reconstruct the interference-plus-noise covariance matrix (INCM) and the pseudo INCM accurately according to their definitions instead of the integration over interference-plus-noise angular sector. These accurate INCM and pseudo INCM ensure that the EINCM is reconstructed precisely. We propose to estimate the EDSSV from the intersection of two extended subspaces. The first extended signal-plus-interference subspace is formed by eigendecomposing the extended SCM, and the second extended DS subspace is constructed by eigendecomposing the extended DS covariance matrix. The estimated EDSSV has a closed-form solution with low complexity, which avoids any optimization software. Simulation results indicate that the proposed WLB provides robust performance against several types of model mismatches.

The rest of this paper is arranged as follows. In Section 2, we describe the noncircular signal array model and introduce the knowledge of optimal WL MDVR beamformer. Section 3 presents the proposed robust WLB with EINCM reconstruction and EDSSV estimation. In Section 4, we carry out the numerical simulations to compare the performance of the proposed robust WLB with the existing WLBs. Finally, We make conclusion in Section 5. For simplicity, we put the definitions of the symbols used in the paper into Table 1 and add a short introduction of the abbreviations used in the paper into Table 2.

\section{Signal model and optimal WL MVDR beamformer}

We consider an array of $N$ antennas receiving $M$ narrowband signals. The array observation vector at the time index $k$ can be modeled as

$$
\mathbf{x}(k)=\mathbf{a}_{1} s_{1}(k)+\mathbf{v}(k),
$$

where $\mathbf{a}_{1}$ and $s_{1}(k)$ are respectively the DSSV and the DS complex waveform, and $\mathbf{v}(k)=\sum_{m=2}^{M} \mathbf{a}_{m} s_{m}(k)+\mathbf{n}(k)$ is the whole interference-plus-noise vector. Here, $\left\{\mathbf{a}_{m}\right\}_{m=2}^{M}$ and $\left\{s_{m}(k)\right\}_{m=2}^{M}$ respectively denote the steering vectors and complex waveforms of interferences, and $\mathbf{n}(k)$ is the noise vector which is assumed to be a zero-mean circularly symmetric Gaussian white process. The DS and interferences are potentially second-order noncircular and statistically independent with each other. We denote the noncircularity coefficient of the $m$ th signal as $\gamma_{m}=\left\langle E\left[s_{m}(k)^{2}\right]\right\rangle / \sigma_{m}^{2}=\left|\gamma_{m}\right| e^{j \phi_{m}}$ for $m=1,2, \cdots, M$, where $\sigma_{m}^{2}=\left\langle E\left[\left|s_{m}(k)\right|^{2}\right]\right\rangle$ is the time-averaged power, $\left|\gamma_{m}\right|$ is the noncircularity rate with $0 \leq\left|\gamma_{m}\right| \leq 1$, and $\phi_{m}$ is the noncircularity phase. Specially, $\left|\gamma_{1}\right|=1$ 
Table 1 The definitions of the symbols used in the paper

\begin{tabular}{|c|c|}
\hline Symbols & Definitions \\
\hline$(\cdot)^{*}$ & Conjugate operator \\
\hline$(\cdot)^{T}$ & Transpose operator \\
\hline$(\cdot)^{H}$ & Hermitian transpose operator \\
\hline$(\cdot)^{-1}$ & Matrix inversion operator \\
\hline$\langle\cdot\rangle$ & Time-averaging operation \\
\hline$E[\cdot]$ & Expectation operator \\
\hline $\mathbf{I}_{N}$ & $N \times N$ identity matrix \\
\hline $\operatorname{diag}\{\cdot\}$ & Diagonalization operator \\
\hline$|\cdot|$ & Absolute value operator \\
\hline$\|\cdot\|$ & Euclidean norm \\
\hline$\cap$ & Set intersection \\
\hline $\operatorname{eig}_{\max }(\cdot)$ & Maximum eigenvalue of a matrix \\
\hline N & Number of array antennas \\
\hline M & Number of narrowband signals \\
\hline k & Time index of snapshots \\
\hline $\mathbf{x}$ & Array observation vector \\
\hline $\mathbf{a}$ & Steering vector \\
\hline$s$ & Complex waveform \\
\hline $\mathbf{v}$ & Whole interference-plus-noise vector \\
\hline$\gamma$ & Noncircularity coefficient \\
\hline$\sigma^{2}$ & Time-averaged power \\
\hline$|\gamma|$ & Noncircularity rate \\
\hline$\phi$ & Noncircularity phase \\
\hline $\mathbf{R}$ & Covariance matrix \\
\hline C & Pseudo covariance matrix \\
\hline$\breve{\mathbf{x}}$ & Extended observation vector \\
\hline$\breve{\mathbf{v}}$ & Extended interference-plus-noise vector \\
\hline $\mathbf{R}_{\breve{X}}$ & Extended covariance matrix \\
\hline$s^{\perp}$ & Orthogonal decomposition of $s$ \\
\hline$\breve{a}$ & Extended steering vector \\
\hline$\breve{\mathbf{v}}_{\gamma}$ & Global noise vector \\
\hline y & WLB output \\
\hline$\breve{\mathbf{w}}$ & WLB weight vector \\
\hline SINR & Output signal-to-interference-plus-noise ratio \\
\hline K & Number of observed snapshots \\
\hline $\bar{\delta}$ & Minimum eigenvalue of $\mathbf{R}_{\hat{x}}$ \\
\hline$\theta$ & Signal direction $\quad x$ \\
\hline$\Theta$ & Signal angular sector \\
\hline$\alpha_{i}$ & Eigenvalue of $\mathbf{R}_{\hat{x}}$ \\
\hline $\mathbf{g}_{i}$ & Eigenvector of $\hat{\mathbf{R}}_{\hat{x}}$ \\
\hline G & Subspace projection matrix \\
\hline$\Gamma$ & Diagonal matrix \\
\hline$\beta$ & Adaptive uncertainty level \\
\hline$\eta$ & Lagrange multiplier \\
\hline$\varrho$ & Threshold constant \\
\hline$\lambda_{i}$ & Eigenvalue of $\mathbf{R}_{\hat{x}}$ \\
\hline $\mathbf{q}_{i}$ & Eigenvector of $\mathbf{R}_{\hat{x}}$ \\
\hline $\mathbf{Q}$ & Matrix containing eigenvectors \\
\hline$\Lambda$ & Diagonal matrix containing eigenvalues \\
\hline$f$ & Projection value \\
\hline Q & Number of principal eigenvectors \\
\hline $\mathbf{F}$ & Extended subspace projection matrix \\
\hline$\epsilon$ & A predefined constant \\
\hline$\Pi$ & Extended subspace \\
\hline$\pi$ & Subspace coefficient vector \\
\hline$p(\theta)$ & Spatial power spectrum \\
\hline$\mu_{l}$ & Eigenvalue of $\breve{\mathbf{R}}_{5}$ \\
\hline $\mathbf{u}_{/}$ & Eigenvector of $\breve{\mathbf{R}}_{s}$ \\
\hline $\mathbf{U}$ & Matrix containing eigenvectors \\
\hline U & Number of principal eigenvectors \\
\hline$\zeta$ & A predefined constant \\
\hline $\boldsymbol{\Omega}$ & Diagonal matrix containing eigenvalues \\
\hline
\end{tabular}


Table 1 The definitions of the symbols used in the paper (Continued)

\begin{tabular}{ll}
\hline Symbols & Definitions \\
\hline $\boldsymbol{\Psi}$ & Extended subspace \\
$\boldsymbol{J}$ & Subspace coefficient vector \\
$J$ & Number of grid points in complementary $\Theta_{1}$ \\
1 & Number of iterations \\
$\mathbf{d}\left(\theta_{p}\right)$ & Coherently scattered paths \\
$\varphi_{p}$ & Scattered path phases \\
$\xi$ & Norm of random steering vector mismatch \\
\hline
\end{tabular}

represents the rectilinear signal whose complex waveform is on a line. The second-order statistics of the noncircular data $\mathbf{x}(k)$ are expressed as

$$
\begin{aligned}
& \mathbf{R}_{x}=\left\langle E\left[\mathbf{x}(k) \mathbf{x}(k)^{H}\right]\right\rangle=\sigma_{1}^{2} \mathbf{a}_{1} \mathbf{a}_{1}^{H}+\mathbf{R}_{v}, \\
& \mathbf{C}_{x}=\left\langle E\left[\mathbf{x}(k) \mathbf{x}(k)^{T}\right]\right\rangle=\sigma_{1}^{2} \gamma_{1} \mathbf{a}_{1} \mathbf{a}_{1}^{T}+\mathbf{C}_{v},
\end{aligned}
$$

where

$$
\begin{aligned}
& \mathbf{R}_{v}=\left\langle E\left[\mathbf{v}(k) \mathbf{v}(k)^{H}\right]\right\rangle=\sum_{m=2}^{M} \sigma_{m}^{2} \mathbf{a}_{m} \mathbf{a}_{m}^{H}+\sigma_{n}^{2} \mathbf{I}_{N}, \\
& \mathbf{C}_{v}=\left\langle E\left[\mathbf{v}(k) \mathbf{v}(k)^{T}\right]\right\rangle=\sum_{m=2}^{M} \sigma_{m}^{2} \gamma_{m} \mathbf{a}_{m} \mathbf{a}_{m}^{T} .
\end{aligned}
$$

Here, $\mathbf{R}_{x}$ and $\mathbf{C}_{x}$ are respectively the theoretical covariance matrix and theoretical pseudo covariance matrix of $\mathbf{x}(k), \mathbf{R}_{v}$ and $\mathbf{C}_{v}$ are respectively the theoretical INCM and theoretical pseudo INCM, and $\sigma_{n}^{2}$ is the noise power.

Table 2 Introduction to the abbreviations used in this paper

\begin{tabular}{ll}
\hline Abbreviations & Introduction \\
\hline WLB & Widely linear beamformer \\
EINCM & Extended interference-plus-noise covariance matrix \\
INCM & Interference-plus-noise covariance matrix \\
EDSSV & Extended desired signal steering vector \\
DS & Desired signal \\
MVDR & Minimum variance distortionless response \\
LCMV & Linearly constrained minimum variance \\
UQPSK & Unbalanced quaternary phase shift keying \\
AM & Amplitude modulated \\
ASK & Amplitude-shift keying \\
BPSK & Binary phase-shift keying \\
WL & Widely linear \\
SNR & Signal-to-noise ratio \\
DSSV & Desired signal steering vector \\
SCM & Sample covariance matrix \\
SSNC & Spatial spectrum of noncircularity coefficient \\
PC & Projection constraint \\
SINR & Signal-to-interference-plus-noise ratio \\
IRCB & Iterative robust Capon beamformer \\
ULA & Uniform linear array \\
INR & Interference-to-noise ratio \\
NC-RCB & Noncircular robust Capon beamformer \\
IQCQP & Iterative Quadratically constrained quadratic programming \\
\hline
\end{tabular}


By stacking $\mathbf{x}(k)$ and its conjugate component, we define the extended observation vector as

$$
\begin{aligned}
\breve{\mathbf{x}}(k) & =\left[\mathbf{x}(k)^{T}, \mathbf{x}(k)^{H}\right]^{T} \\
& =s_{1}(k) \mathbf{b}_{1}+s_{1}(k)^{*} \mathbf{c}_{1}+\breve{\mathbf{v}}(k),
\end{aligned}
$$

where $\mathbf{b}_{1}=\left[\mathbf{a}_{1}^{T}, \mathbf{0}_{N}^{T}\right]^{T}, \mathbf{c}_{1}=\left[\mathbf{0}_{N}^{T}, \mathbf{a}_{1}^{H}\right]^{T}$, and $\breve{\mathbf{v}}(k)=\left[\mathbf{v}(k)^{T}, \mathbf{v}(k)^{H}\right]^{T}$. The extended covariance matrix of $\breve{\mathbf{x}}(k)$ is denoted as

$$
\mathbf{R}_{\breve{x}}=\left\langle E\left[\breve{\mathbf{x}}(k) \breve{\mathbf{x}}(k)^{H}\right]\right\rangle=\left[\begin{array}{ll}
\mathbf{R}_{x} & \mathbf{C}_{x} \\
\mathbf{C}_{x}^{*} & \mathbf{R}_{x}^{*}
\end{array}\right] .
$$

To further utilize the noncircularity of the DS, we orthogonally decompose $s_{1}(k)^{*}$ as

$$
s_{1}(k)^{*}=\gamma_{1}^{*} s_{1}(k)+\left[\sigma_{1}^{2}\left(1-\left|\gamma_{1}\right|^{2}\right)\right]^{1 / 2} s_{1}^{\perp}(k),
$$

where $\left\langle E\left[s_{1}(k) s_{1}^{\perp}(k)^{*}\right]\right\rangle=0$ and $\left\langle E\left[\left|s_{1}^{\perp}(k)\right|^{2}\right]\right\rangle=1$. In this way, we rewrite $\breve{\mathbf{x}}(k)$ in (6) as

$$
\breve{\mathbf{x}}(k)=s_{1}(k) \breve{\mathbf{a}}_{1}+\breve{\mathbf{v}}_{\gamma}(k),
$$

with

$$
\begin{aligned}
& \breve{\mathbf{a}}_{1}=\mathbf{b}_{1}+\gamma_{1}^{*} \mathbf{c}_{1}=\left[\mathbf{a}_{1}^{T}, \gamma_{1}^{*} \mathbf{a}_{1}^{H}\right]^{T}, \\
& \breve{\mathbf{v}}_{\gamma}(k)=s_{1}^{\perp}(k)\left[\sigma_{1}^{2}\left(1-\left|\gamma_{1}\right|^{2}\right)\right]^{1 / 2} \mathbf{c}_{1}+\breve{\mathbf{v}}(k),
\end{aligned}
$$

where $\breve{\mathbf{a}}_{1}$ is the noncircular EDSSV and $\breve{\mathbf{v}}_{\gamma}(k)$ is the global noise vector for $\breve{\mathbf{x}}(k)$. The WLB output is denoted as

$$
y(k)=\breve{\mathbf{w}}^{H} \breve{\mathbf{x}}(k)=s_{1}(k) \breve{\mathbf{w}}^{H} \breve{\mathbf{a}}_{1}+\breve{\mathbf{w}}^{H} \breve{\mathbf{v}}_{\gamma}(k),
$$

where $\breve{\mathbf{w}}$ is the WLB weight vector. The optimal WL MVDR beamformer can be designed by solving [22]

$$
\min _{\breve{\mathbf{w}}} \breve{\mathbf{w}}^{H} \mathbf{R}_{\breve{v}_{\gamma}} \breve{\mathbf{w}} \quad \text { s.t. } \quad \breve{\mathbf{w}}^{H} \breve{\mathbf{a}}_{1}=1,
$$

where

$$
\mathbf{R}_{\breve{v}_{\gamma}}=\left\langle E\left[\breve{\mathbf{v}}_{\gamma}(k) \breve{\mathbf{v}}_{\gamma}(k)^{H}\right]\right\rangle=\left[\begin{array}{ll}
\mathbf{R}_{v} & \mathbf{C}_{v} \\
\mathbf{C}_{v}^{*} & \mathbf{R}_{v}^{*}
\end{array}\right],
$$

denotes the theoretical EINCM. The solution of (13) is given by

$$
\breve{\mathbf{w}}_{\text {MVDR }}=\left[\breve{\mathbf{a}}_{1}^{H} \mathbf{R}_{\breve{v}_{\gamma}}^{-1} \breve{\mathbf{a}}_{1}\right]^{-1} \mathbf{R}_{\breve{v}_{\gamma}}^{-1} \breve{\mathbf{a}}_{1} .
$$

The output signal-to-interference-plus-noise ratio (SINR) of a WLB is defined as

$$
\operatorname{SINR}=\frac{\sigma_{1}^{2}\left|\breve{\mathbf{w}}^{H} \breve{\mathbf{a}}_{1}\right|^{2}}{\breve{\mathbf{w}}^{H} \mathbf{R}_{\breve{v}_{\gamma}} \breve{\mathbf{w}}}
$$

However, the theoretical $\mathbf{R}_{\breve{v}_{\gamma}}$ and $\breve{\mathbf{a}}_{1}$ are unfortunately not available in practice. In such cases, one may approximate $\mathbf{R}_{\breve{v}_{\gamma}}$ as the following extended SCM

$$
\mathbf{R}_{\hat{x}}=\frac{1}{K} \sum_{k=1}^{K} \hat{\tilde{\mathbf{x}}}(k) \hat{\tilde{\mathbf{x}}}(k)^{H}=\left[\begin{array}{ll}
\mathbf{R}_{\hat{x}} & \mathbf{C}_{\hat{x}} \\
\mathbf{C}_{\hat{x}}^{*} & \mathbf{R}_{\hat{x}}^{*}
\end{array}\right],
$$

where $\mathbf{R}_{\hat{x}}=(1 / K) \sum_{k=1}^{K} \hat{\mathbf{x}}(k) \hat{\mathbf{x}}(k)^{H}$ and $\mathbf{C}_{\hat{x}}=(1 / K) \sum_{k=1}^{K} \hat{\mathbf{x}}(k) \hat{\mathbf{x}}(k)^{T}$ respectively represent the SCM and the pseudo SCM that are obtained using $K$ observed snapshots. The unknown vector $\breve{\mathbf{a}}_{1}$ is usually approximated by the presumed EDSSV $\overline{\mathbf{a}}_{1}$ with the exactly 
known DS noncircularity coefficient $\gamma_{1}$. To facilitate the implementation of the WLB, the authors in [26] propose to estimate the DS noncircularity coefficient as

$$
\bar{\gamma}_{1}=-\frac{\mathbf{a}_{1}^{H} \mathbf{E} \mathbf{a}_{1}^{*}}{\mathbf{a}_{1}^{H} \mathbf{D} \mathbf{a}_{1}} \cdot \frac{\mathbf{a}_{1}^{H} \mathbf{a}_{1}}{\mathbf{a}_{1}^{H}\left(\mathbf{I}_{N}-\bar{\delta} \mathbf{R}_{\hat{x}}^{-1}\right) \mathbf{a}_{1}},
$$

where $\mathbf{D}=\left(\mathbf{R}_{\hat{x}}-\mathbf{C}_{\hat{x}} \mathbf{R}_{\hat{x}}^{*-1} \mathbf{C}_{\hat{x}}^{*}\right)^{-1}, \mathbf{E}=-\mathbf{D} \mathbf{C}_{\hat{x}} \mathbf{R}_{\hat{x}}^{*-1}$, and $\bar{\delta}$ is the minimum eigenvalue of $\mathbf{R}_{\hat{x}}$.

\section{Proposed robust WLB}

From (15), we observe that the extended weight vector of a WLB is a function of the EINCM and the EDSSV. In this section, we use this dependency to design a lowcomplexity robust WLB by reconstructing precise EINCM and estimating accurate EDSSV.

\subsection{EINCM reconstruction}

According to (14), reconstructing the EINCM requires the INCM and pseudo INCM. From (4), the INCM is related to the powers and steering vectors of interferences and the noise power. From (5), the pseudo INCM is related to the powers, noncircularity coefficients, and steering vectors of interferences. Therefore, we should estimate the steering vectors of interferences first. Then, we estimate the powers and noncircularity coefficients of interferences and estimate the noise power. Finally, we reconstruct the INCM, pseudo INCM, and EINCM.

The rough directions of all signals can be easily determined by the beampattern nulling method [7] or the low-resolution direction-of-arrival estimation method [33]. We respectively denote the rough directions and angular sectors of all signals as $\left\{\bar{\theta}_{m}\right\}_{m=1}^{M}$ and $\left\{\Theta_{m}\right\}_{m=1}^{M}$. The steering vector corresponding to the signal with direction $\bar{\theta}_{m}$ is denoted as $\overline{\mathbf{a}}_{m}$ for $m=1,2, \cdots, M$.

It is obvious that the steering vector mismatches exist in $\left\{\overline{\mathbf{a}}_{m}\right\}_{m=1}^{M}$ due to the direction mismatches in $\left\{\bar{\theta}_{m}\right\}_{m=1}^{M}$. Hence, we have to correct the steering vectors $\left\{\overline{\mathbf{a}}_{m}\right\}_{m=1}^{M}$. In [34], the IRCB can only estimate the DSSV. Here, we extend the IRCB to not only correct the DSSV but also correct the interference steering vectors. We eigendecompose the $\operatorname{SCM} \mathbf{R}_{\hat{x}}$ as

$$
\mathbf{R}_{\hat{x}}=\sum_{i=1}^{N} \alpha_{i} \mathbf{g}_{i} \mathbf{g}_{i}^{H}=\mathbf{G}_{s}^{H} \boldsymbol{\Gamma}_{s} \mathbf{G}_{s}+\mathbf{G}_{n}^{H} \boldsymbol{\Gamma}_{n} \mathbf{G}_{n},
$$

where $\alpha_{1} \geq \alpha_{2} \geq \cdots \geq \alpha_{N}$ are the eigenvalues of $\mathbf{R}_{\hat{x}}$ and $\mathbf{g}_{i}$ is the eigenvector associated with the eigenvalue $\alpha_{i} . \mathbf{G}_{s}=\left[\mathbf{g}_{1}, \mathbf{g}_{2}, \cdots, \mathbf{g}_{M}\right]$ spans the signal-plus-interference subspace and $\mathbf{G}_{n}=\left[\mathbf{g}_{M+1}, \mathbf{g}_{M+2}, \cdots, \mathbf{g}_{N}\right]$ spans the noise subspace. $\boldsymbol{\Gamma}_{s}=\operatorname{diag}\left\{\alpha_{1}, \alpha_{2}, \cdots, \alpha_{M}\right\}$ and $\boldsymbol{\Gamma}_{n}=\operatorname{diag}\left\{\alpha_{M+1}, \alpha_{M+1}, \cdots, \alpha_{N}\right\}$ are the diagonal matrices. We set the iterative initial values as

$$
\hat{\mathbf{a}}_{m}^{0}=\sqrt{N} \overline{\mathbf{a}}_{m} /\left\|\overline{\mathbf{a}}_{m}\right\|, m=1,2, \cdots, M .
$$

The iteration process of IRCB for the $m$ th signal can be given by

$$
\beta_{m}^{i+1}=\left\|\left(\frac{\sqrt{N}}{\left\|\mathbf{G}_{s} \mathbf{G}_{s}^{H} \hat{\mathbf{a}}_{m}^{i}\right\|}-2\right) \mathbf{G}_{s} \mathbf{G}_{s}^{H} \hat{\mathbf{a}}_{m}^{i}+\hat{\mathbf{a}}_{m}^{i}\right\|^{2},
$$




$$
\begin{aligned}
& \min _{\hat{\mathbf{a}}_{m}^{i+1}}\left(\hat{\mathbf{a}}_{m}^{i+1}\right)^{H} \mathbf{R}_{\hat{x}}^{-1} \hat{\mathbf{a}}_{m}^{i+1} \quad \text { s.t. }\left\|\hat{\mathbf{a}}_{m}^{i+1}-\hat{\mathbf{a}}_{m}^{i}\right\| \leq \beta_{m}^{i+1}, \\
& \hat{\mathbf{a}}_{m}^{i+1}=\sqrt{N} \hat{\mathbf{a}}_{m}^{i+1} /\left\|\hat{\mathbf{a}}_{m}^{i+1}\right\|,
\end{aligned}
$$

where $\beta_{m}^{i+1}$ is the adaptive uncertainty level of the $m$ th signal in the $(i+1)$ th iteration. The solution of (22) is expressed as

$$
\hat{\mathbf{a}}_{m}^{i+1}=\hat{\mathbf{a}}_{m}^{i}-\left(\mathbf{I}_{N}+\eta \mathbf{R}_{\hat{x}}\right)^{-1} \hat{\mathbf{a}}_{m}^{i},
$$

where $\eta$ is the Lagrange multiplier which is obtained as the solution to the following equation

$$
\left\|\left(\mathbf{I}_{N}+\eta \mathbf{R}_{\hat{x}}\right)^{-1} \hat{\mathbf{a}}_{m}^{i}\right\|^{2}=\beta_{m}^{i+1} .
$$

The purpose of (23) is to obtain a maximal invariant and to avoid the norm ambiguity. The iteration process in (21)-(23) will stop when $\beta_{m}^{i}-\beta_{m}^{i+1} \leq \varrho$, where $\varrho$ is a threshold constant. After the whole iteration process is completed, we denote the final steering vector estimate of the $m$ th signal as $\hat{\mathbf{a}}_{m}$ for $m=1,2, \cdots, M$.

By using the covariance fitting approach, the power of the $m$ th signal is obtained from

$$
\hat{\sigma}_{m}^{2}=\frac{1}{\hat{\mathbf{a}}_{m}^{H} \mathbf{R}_{\hat{x}}^{-1} \hat{\mathbf{a}}_{m}}, \quad m=1,2, \cdots, M .
$$

According to (4), we reconstruct the INCM as

$$
\hat{\mathbf{R}}_{v}=\sum_{m=2}^{M} \hat{\sigma}_{m}^{2} \hat{\mathbf{a}}_{m} \hat{\mathbf{a}}_{m}^{H}+\alpha_{N} \mathbf{I}_{N},
$$

where $\alpha_{N}$ is the minimum eigenvalue of $\mathbf{R}_{\hat{x}}$ which is treated as the estimated noise power. The noncircularity coefficient estimator (18) can only estimate the DS noncircularity coefficient. Here, we extend (18) to estimate the noncircularity coefficients of DS and interferences. By replacing $\mathbf{a}_{1}$ in (18) with $\hat{\mathbf{a}}_{m}$, we calculate the noncircularity coefficient of the $m$ th noncircular signal as

$$
\hat{\gamma}_{m}=-\frac{\hat{\mathbf{a}}_{m}^{H} \mathbf{E} \hat{\mathbf{a}}_{m}^{*}}{\hat{\mathbf{a}}_{m}^{H} \mathbf{D} \hat{\mathbf{a}}_{m}} \cdot \frac{\hat{\mathbf{a}}_{m}^{H} \hat{\mathbf{a}}_{m}}{\hat{\mathbf{a}}_{m}^{H}\left(\mathbf{I}_{N}-\bar{\delta} \mathbf{R}_{\hat{x}}^{-1}\right) \hat{\mathbf{a}}_{m}},
$$

for $m=1,2, \cdots, M$. According to (5), we reconstruct the pseudo INCM as

$$
\hat{\mathbf{C}}_{v}=\sum_{m=2}^{M} \hat{\sigma}_{m}^{2} \hat{\gamma}_{m} \hat{\mathbf{a}}_{m} \hat{\mathbf{a}}_{m}^{T} .
$$

By using the reconstructed INCM $\hat{\mathbf{R}}_{v}$ and the reconstructed pseudo INCM $\hat{\mathbf{C}}_{v}$, we can reconstruct the EINCM as

$$
\hat{\mathbf{R}}_{\breve{v}_{\gamma}}=\left[\begin{array}{cc}
\hat{\mathbf{R}}_{v} & \hat{\mathbf{C}}_{v} \\
\hat{\mathbf{C}}_{v}^{*} & \hat{\mathbf{R}}_{v}^{*}
\end{array}\right] .
$$

\subsection{EDSSV estimation}

By substituting $\hat{\mathbf{a}}_{1}$ and $\hat{\gamma}_{1}$ into (10), we can compute the EDSSV as

$$
\breve{\hat{\mathbf{a}}}_{1}=\left[\hat{\mathbf{a}}_{1}^{T}, \hat{\gamma}_{1}^{*} \hat{\mathbf{a}}_{1}^{H}\right]^{T} \text {. }
$$

However, the EDSSV $\breve{\hat{\mathbf{a}}}_{1}$ may have relatively large error because both $\hat{\mathbf{a}}_{1}$ and $\hat{\gamma}_{1}$ have estimation errors. Hence, we have to further improve the accuracy of the EDSSV $\breve{\hat{\mathbf{a}}}_{1}$. The extended SCM $\mathbf{R}_{\hat{x}}$ can be eigendecomposed as 


$$
\mathbf{R}_{\hat{x}}=\sum_{i=1}^{2 N} \lambda_{i} \mathbf{q}_{i} \mathbf{q}_{i}^{H}=\mathbf{Q}^{H} \boldsymbol{\Lambda} \mathbf{Q},
$$

where $\lambda_{1} \geq \lambda_{2} \geq \cdots \geq \lambda_{2 N}$ are the eigenvalues of $\mathbf{R}_{\hat{x}}, \mathbf{q}_{i}$ is the eigenvector associated with the eigenvalue $\lambda_{i}, \mathbf{Q}=\left[\mathbf{q}_{1}, \mathbf{q}_{2}, \cdots, \mathbf{q}_{2 N}\right]$ and $\boldsymbol{\Lambda}=\operatorname{diag}\left\{\lambda_{1}, \lambda_{2}, \cdots, \lambda_{2 N}\right\}$. In [35], the signal-plus-interference subspace is formed by projecting the presumed DSSV onto the eigenvectors of SCM. Here, we focus on forming the extended signal-plus-interference subspace. We project the EDSSV $\breve{\hat{a}}_{1}$ onto the $\mathbf{q}_{i}, i=1,2, \cdots, 2 N$ as

$$
f(i)=\left|\mathbf{q}_{i}^{H} \breve{\hat{\mathbf{a}}}_{1}\right|, \quad i=1,2, \cdots, 2 N .
$$

We sort $\{f(i)\}_{i=1}^{2 N}$ in descending order as $f(2 N)>f(2 N-1)>\cdots>f(1)$ and sort its corresponding eigenvectors as $\left[\mathbf{q}_{2 N}, \mathbf{q}_{2 N-1}, \cdots, \mathbf{q}_{1}\right]$. By choosing $Q$ principal eigenvectors, the extended signal-plus-interference subspace projection matrix is constructed as

$$
\mathbf{F}=\left[\mathbf{q}_{2 N}, \mathbf{q}_{2 N-1}, \cdots, \mathbf{q}_{2 N-Q+1}\right],
$$

where $Q$ is the minimum integer satisfying the following relationship

$$
\frac{f(2 N)+f(2 N-1)+\cdots+f(2 N-Q+1)}{2 N}>\epsilon,
$$

where $0<\epsilon<1$ is a predefined constant. It is clear that the actual EDSSV should lie in the subspace spanned by the columns of $\mathbf{F}$, which is denoted as

$$
\breve{\mathbf{a}}_{1} \in \boldsymbol{\Pi}=[\breve{\mathbf{a}}: \breve{\mathbf{a}}=\mathbf{F} \boldsymbol{\pi}],
$$

where $\pi$ is the subspace coefficient vector.

By integrating over DS angular sector $\Theta_{1}$, we construct the DS covariance matrix as

$$
\mathbf{R}_{s}=\int_{\Theta_{1}} p(\theta) \mathbf{a}(\theta) \mathbf{a}(\theta)^{H} \mathrm{~d} \theta, \quad \theta \in \Theta_{1},
$$

where $p(\theta)$ can be chosen as $1 /\left[\mathbf{a}(\theta)^{H} \mathbf{R}_{\hat{x}}^{-1} \mathbf{a}(\theta)\right][36]$ or $1 /\left[\mathbf{a}(\theta)^{H} \mathbf{R}_{\hat{x}}^{-2} \mathbf{a}(\theta)\right]$ [37]. The corresponding pseudo DS covariance matrix is constructed as

$$
\mathbf{C}_{s}=\int_{\Theta_{1}} p(\theta) \bar{\gamma}(\theta) \mathbf{a}(\theta) \mathbf{a}(\theta)^{T} \mathrm{~d} \theta, \quad \theta \in \Theta_{1},
$$

where $\bar{\gamma}(\theta)$ is obtained by replacing $\mathbf{a}_{1}$ with $\mathbf{a}(\theta)$ in (18), which is represented as

$$
\bar{\gamma}(\theta)=-\frac{\mathbf{a}(\theta)^{H} \mathbf{E a}(\theta)^{*}}{\mathbf{a}(\theta)^{H} \mathbf{D a}(\theta)} \cdot \frac{\mathbf{a}(\theta)^{H} \mathbf{a}(\theta)}{\mathbf{a}(\theta)^{H}\left(\mathbf{I}_{N}-\bar{\delta} \mathbf{R}_{\hat{x}}^{-1}\right) \mathbf{a}(\theta)} .
$$

By using $\mathbf{R}_{s}$ and $\mathbf{C}_{s}$, the extended DS covariance matrix can be expressed as

$$
\breve{\mathbf{R}}_{s}=\left[\begin{array}{cc}
\mathbf{R}_{s} & \mathbf{C}_{s} \\
\mathbf{C}_{s}^{*} & \mathbf{R}_{s}^{*}
\end{array}\right] \text {. }
$$

We perform eigendecomposition on $\breve{\mathbf{R}}_{s}$ as

$$
\breve{\mathbf{R}}_{s}=\sum_{l=1}^{2 N} \mu_{l} \mathbf{u}_{l} \mathbf{u}_{l}^{H}=\mathbf{U}_{s} \boldsymbol{\Omega}_{s} \mathbf{U}_{s}^{H}+\mathbf{U}_{n} \boldsymbol{\Omega}_{n} \mathbf{U}_{n}^{H},
$$

where $\mu_{1} \geq \mu_{2} \geq \cdots \geq \mu_{2 N}$ are the eigenvalues of $\breve{\mathbf{R}}_{s}, \mathbf{u}_{l}$ is the eigenvector associated with the eigenvalue $\mu_{l}$. $\mathbf{U}_{s}=\left[\mathbf{u}_{1}, \mathbf{u}_{2}, \cdots, \mathbf{u}_{U}\right]$ consists of $U$ principal eigenvectors of $\breve{\mathbf{R}}_{s}$, where $U$ is the minimum integer satisfying

$$
\frac{\sum_{l=1}^{U}\left|\mu_{l}\right|}{\sum_{l=1}^{2 N}\left|\mu_{l}\right|}>\zeta
$$


with a predefined constant $0<\zeta<1 . \mathbf{U}_{n}=\left[\mathbf{u}_{U+1}, \mathbf{u}_{U+2}, \cdots, \mathbf{u}_{2 N}\right]$ consists of the remaining eigenvectors. $\boldsymbol{\Omega}_{s}=\operatorname{diag}\left\{\mu_{1}, \mu_{2}, \cdots, \mu_{U}\right\}$ contains $U$ largest eigenvalues and $\boldsymbol{\Omega}_{n}=\operatorname{diag}\left\{\mu_{U+1}, \mu_{U+2}, \cdots, \mu_{2 N}\right\}$ contains the remaining eigenvalues. Obviously, the actual EDSSV also lies in the subspace spanned by the columns of $\mathbf{U}_{s}$, which is given by

$$
\breve{\mathbf{a}}_{1} \in \boldsymbol{\Psi}=\left[\breve{\mathbf{a}}: \breve{\mathbf{a}}=\mathbf{U}_{s} \boldsymbol{\psi}\right],
$$

where $\psi$ is the subspace coefficient vector.

From (36) and (43), we can conclude that the actual EDSSV should lie in the intersection of $\Pi$ and $\boldsymbol{\Psi}$, i.e. $\breve{\mathbf{a}}_{1} \in \Pi \cap \boldsymbol{\Psi}$. We can use the alternating projection approach to update $\breve{\mathbf{a}}_{1}^{j+1}$ in the $(j+1)$ th iteration as

$$
\breve{\mathbf{a}}_{1}^{j+1}=\mathbf{L}_{a} \mathbf{L}_{b} \breve{\mathbf{a}}_{1}^{j} \text {, }
$$

where $\breve{\mathbf{a}}_{1}^{0}=\breve{\hat{\mathbf{a}}}_{1}, \mathbf{L}_{a}=\mathbf{F} \mathbf{F}^{H}$ and $\mathbf{L}_{b}=\mathbf{U}_{s} \mathbf{U}_{s}^{H}$. As $j \rightarrow \infty, \breve{\mathbf{a}}_{1}^{j}$ should converge to the actual EDSSV [38-40]. The maximum eigenvalue of $\mathbf{L}_{a} \mathbf{L}_{b}$ is one, which is proven as [41]

$$
\begin{aligned}
\operatorname{eig}_{\max }\left(\mathbf{L}_{a} \mathbf{L}_{b}\right) & \leq \max _{\substack{\mathbf{h} \\
\mathbf{h}^{H} \mathbf{h}=1}} \mathbf{h}^{H} \mathbf{L}_{a} \mathbf{h} \operatorname{eig}_{\max }\left(\mathbf{L}_{b}\right) \\
& =\max _{\substack{\mathbf{h} \\
\mathbf{h}_{\mathbf{h}=1}}} \frac{\mathbf{h}^{H} \mathbf{L}_{a} \mathbf{h}}{\mathbf{h}^{H} \mathbf{h}} \\
& =\operatorname{eig}_{\max }\left(\mathbf{L}_{a}\right)=1 .
\end{aligned}
$$

Therefore, the EDSSV can be computed as

$$
\breve{\mathbf{r}}=\sqrt{2 N} \Upsilon\left(\mathbf{L}_{a} \mathbf{L}_{b}\right),
$$

where $\Upsilon\left(\mathbf{L}_{a} \mathbf{L}_{b}\right)$ represents the eigenvector associated with the maximum eigenvalue of $\mathbf{L}_{a} \mathbf{L}_{b}$. When we divide $\breve{\mathbf{r}} \in \mathbb{C}^{2 N \times 1}$ into two subvectors as $\breve{\mathbf{r}}=\left[\mathbf{r}_{1}^{T}, \mathbf{r}_{2}^{T}\right]^{T}, \mathbf{r}_{1}, \mathbf{r}_{2} \in \mathbb{C}^{N \times 1}$ should satisfy the special structure in (10), i.e. , $\mathbf{r}_{2}=\gamma_{r}^{*} \mathbf{r}_{1}^{*}$. However, this relationship may not be satisfied because of the existence of error. To further correct $\breve{\mathbf{r}}$, we have to solve the following problem

$$
\min _{\gamma_{r}^{*}}\left\|\mathbf{r}_{2}-\gamma_{r}^{*} \mathbf{r}_{1}\right\|^{2}
$$

The solution of (47) is given by

$$
\hat{\gamma}_{r}^{*}=\mathbf{r}_{2}^{H} \mathbf{r}_{1}^{*} /\left(\mathbf{r}_{1}^{T} \mathbf{r}_{1}^{*}\right) .
$$

In such case, $\breve{\mathbf{r}}$ can be corrected as $\hat{\mathbf{r}}=\left[\mathbf{r}_{1}^{T}, \hat{\gamma}_{r}^{*} \mathbf{r}_{1}^{H}\right]^{T}$. Finally, the EDSSV is corrected as

$$
\hat{\hat{\mathbf{a}}}_{1}=\sqrt{2 N \hat{\mathbf{r}}} /\|\hat{\mathbf{r}}\| \text {. }
$$

\subsection{Extended weight vector calculation}

By combining the EINCM $\hat{\mathbf{R}}_{\breve{v}_{\gamma}}$ and the EDSSV $\hat{\hat{\mathbf{a}}}_{1}$, the extended weight vector of the proposed robust WLB is calculated as

$$
\hat{\tilde{\mathbf{w}}}=\left[\hat{\hat{\mathbf{a}}}_{1}^{H} \hat{\mathbf{R}}_{\breve{v}_{\gamma}}^{-1} \hat{\hat{\mathbf{a}}}_{1}\right]^{-1} \hat{\mathbf{R}}_{\breve{v}_{\gamma}}^{-1} \hat{\hat{\mathbf{a}}}_{1} .
$$

The proposed WL beamforming algorithm is summarized in Algorithm 1. 


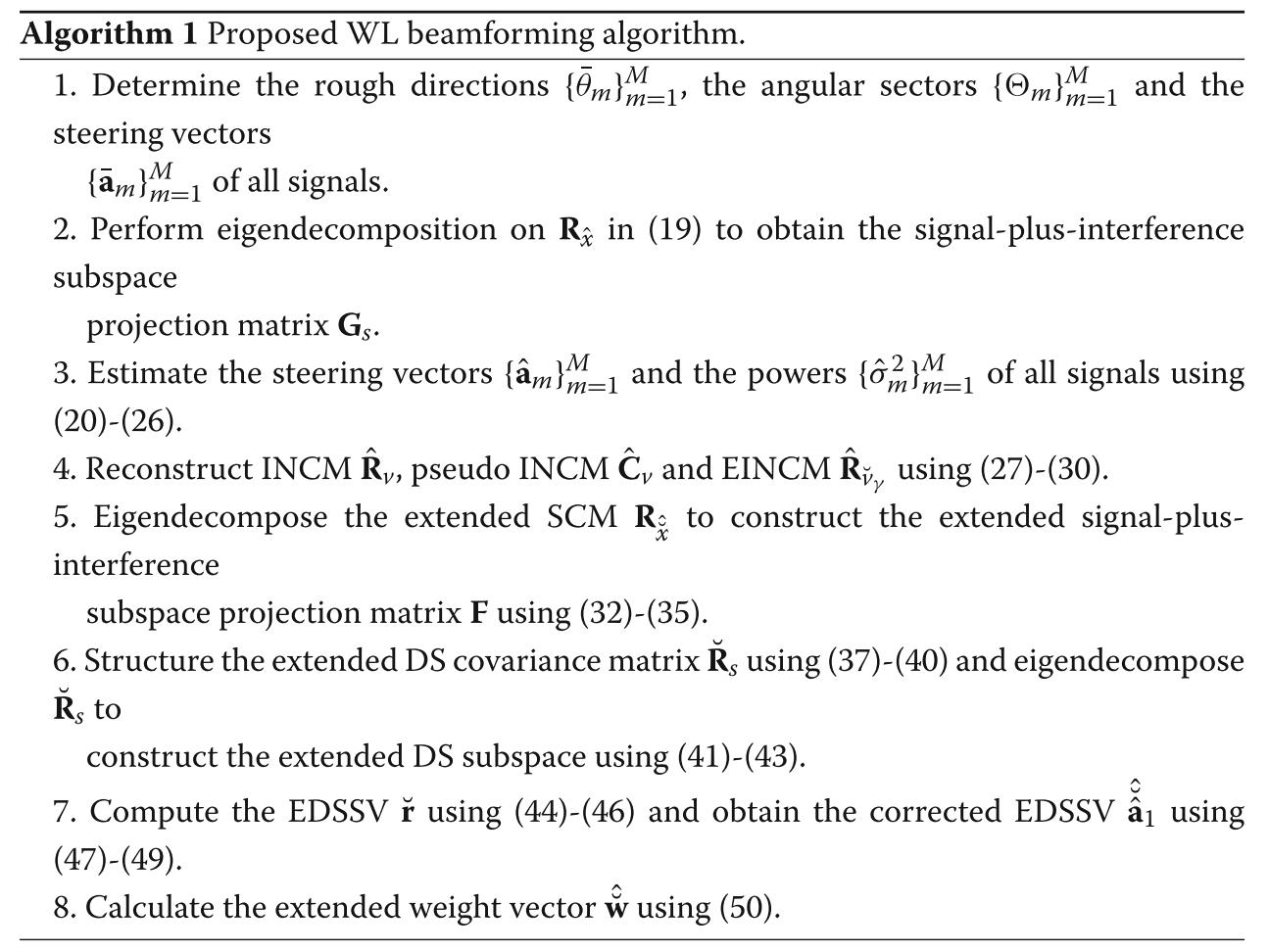

\subsection{Computational complexity}

In the proposed WLB, the main computational cost of EINCM reconstruction lies in the eigendecomposition of $\mathbf{R}_{\hat{x}}$ with a complexity of $\mathcal{O}\left(N^{3}\right)$, and the EDSSV estimation has a complexity of $\mathcal{O}\left(8 N^{3}\right)$ dominated by the eigendecomposition of $\mathbf{R}_{\hat{\grave{x}}}$ from the standpoint of computational complexity. Therefore, the main computational complexity of the proposed WLB is $\mathcal{O}\left(8 N^{3}\right)$. The main computational complexity of the WL-SSNC beamformer [30] is $\mathcal{O}\left(\max \left\{J N^{2}, N^{3.5}+(2 N)^{3.5}\right\}\right)$, where EINCM reconstruction costs a complexity of $\mathcal{O}\left(J N^{2}\right)$ with $J$ grid points in the complement angular sector of $\Theta_{1}$ and EDSSV estimation costs a complexity of $\mathcal{O}\left(N^{3.5}+(2 N)^{3.5}\right)$. The main computational cost of the WL-PC beamformer [31] arises from solving the convex optimization problem with a complexity $\mathcal{O}\left((2 N)^{3.5}\right)$. The computational complexity of the WL via iterative quadratically constrained quadratic programming (WL-IQCQP) beamformer [32] is $\mathcal{O}\left(\max \left\{J N^{2}, I N^{3.5}\right\}\right)$, where $I$ is the number of iterations in [32]. As it can be seen, the proposed WLB has lower computational complexity than WL-SSNC beamformer [30], WL-PC beamformer [31], and WL-IQCQP beamformer [32].

We provide a visual comparison of computational complexity by plotting the flops curves of WL-SSNC beamformer [30], WL-PC beamformer [31], WL-IQCQP beamformer [32], and the proposed WLB. We set $J$ to be 170 for the WL-SSNC beamformer [30] and the WL-IQCQP beamformer [32]. $I=3$ is selected for the WL-IQCQP beamformer [32]. Figure 1 plots the flops curves of all the above WLBs versus the number of array antennas $N$ ranging from 10 to 100 . Obviously, we can see that the proposed WLB has the lowest computational complexity among the examined WLBs. 


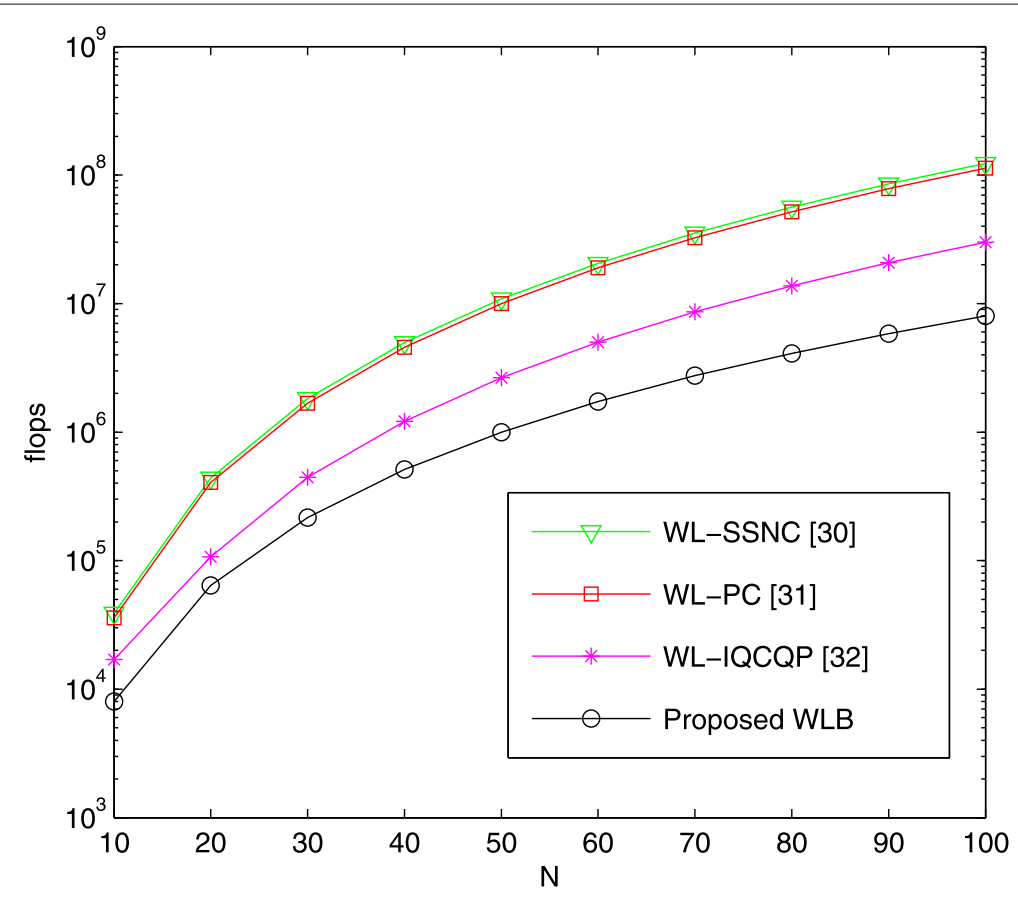

Fig. 1 Comparison of computational complexity. Flops curves versus the number of antennas $N$

\section{Simulation results}

In this section, we perform simulation examples to examine the performance of the proposed robust WLB and compare it with other WLBs. We consider a uniform linear array (ULA) of $N=10$ omnidirectional sensors with inter-element spacing of half a wavelength. Three BPSK signals including one desired signal and two interferences impinge on the considered array. The DS arrives at the considered array from $\bar{\theta}_{1}=5^{\circ}$ with the noncircularity phase $60^{\circ}$. Two interferences come from $-30^{\circ}$ and $60^{\circ}$ with the noncircularity phases $-120^{\circ}$ and $150^{\circ}$, respectively. The interference-to-noise ratio (INR) is set as $10 \mathrm{~dB}$. The additive noise is a complex circularly symmetric Gaussian white process with zero mean and unit variance. Each point in the curves is an average of 200 Monte Carlo trials.

The proposed WLB is compared to the WL-RCB [25], the noncircular robust Capon beamformer (NC-RCB) [27], the WL-SSNC beamformer [30], the WL-PC beamformer [31], and the WL-IQCQP beamformer [32]. For the proposed WLB, $\varrho=10^{-5}, \epsilon=0.9$ and $\zeta=0.7$ are exploited. The values $\varepsilon_{\gamma}=0.1$ and $\varepsilon_{a}=0.3 N$ are used for the WL-RCB beamformer [25]. $\tilde{\varepsilon}=0.6$ is chosen for the NC-RCB beamformer [27]. We set the DS angular sector as $\Theta_{1}=\left[0^{\circ}, 10^{\circ}\right]$ for the WL-SSNC [30], the WL-PC [31], the WL-IQCQP [32], and the proposed WLB. $\rho=0.9$ is used for the WL-PC beamformer [31]. $\varpi$ is set to be $10^{-4}$ for the WL-IQCQP beamformer [32].

Example 1 We investigate the effect of fixed look direction mismatch on the beamforming performance. The presumed DS direction is set as $5^{\circ}$ while the actual DS direction is $2^{\circ}$, which means that the DS look direction mismatch is fixed at $3^{\circ}$. The output SINR of the aforementioned WLBs versus the input SNR with the fixed snapshots number $K=100$ 


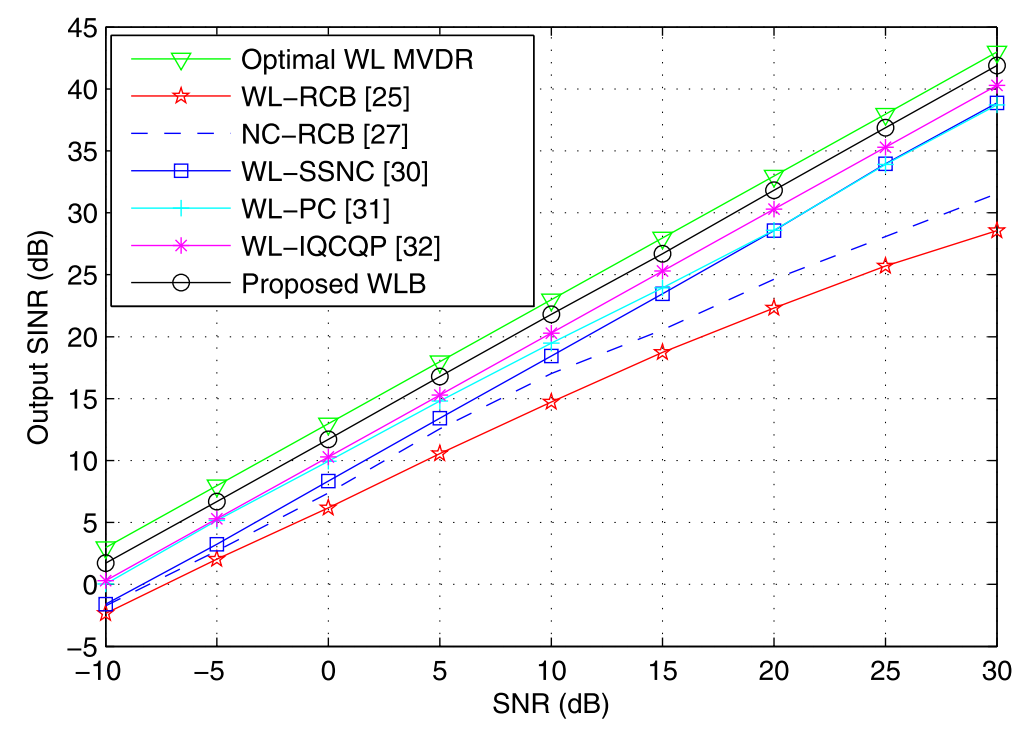

Fig. 2 Example 1. Output SINR of all WLBs versus the input SNR

is depicted in Fig. 2. It can be observed that the WL-SSNC, the WL-IQCQP and the proposed WLB have robust performance because these three WLBs remove the DS component from the extended SCM by reconstructing EINCM. The proposed WLB has the best performance among the tested WLBs because the proposed WLB reconstruct precise EINCM. Figure 3 shows the output SINR of the examined WLBs versus the number of snapshots with fixed $S N R=20 \mathrm{~dB}$. We can see that the proposed WLB always achieves near optimal performance and it outperforms the other WLBs.

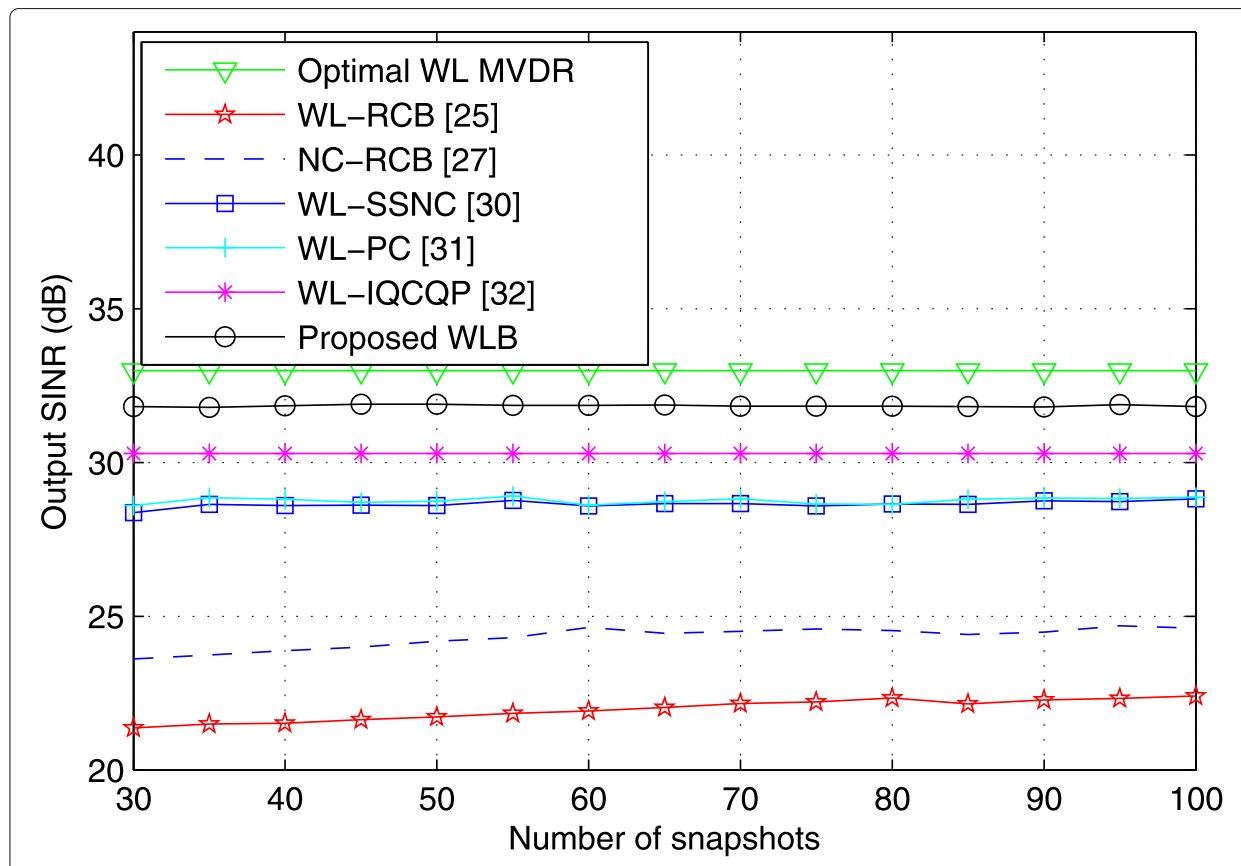

Fig. 3 Example 1. Output SINR of all WLBs versus the number of snapshots $K$ 


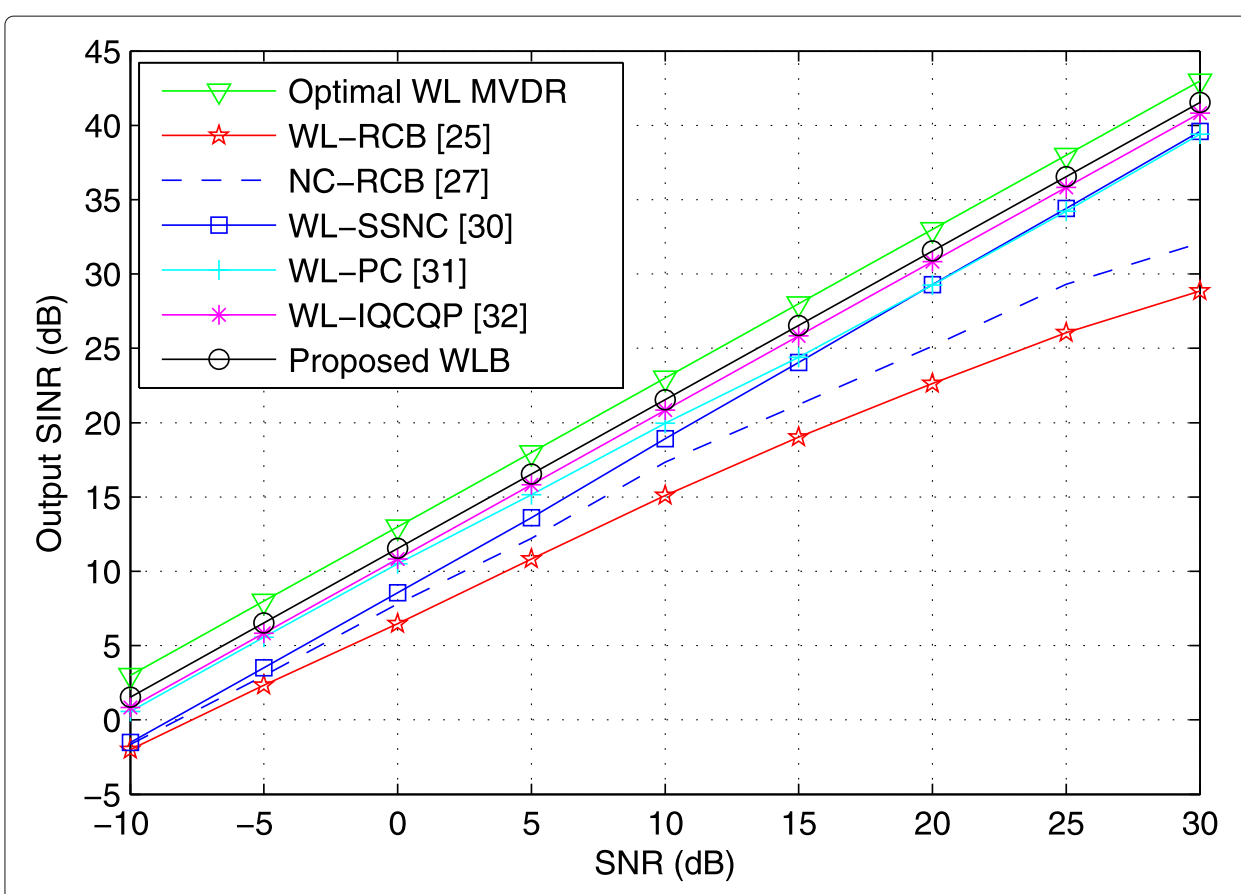

Fig. 4 Example 2. Output SINR of all WLBs versus the input SNR

Example 2 We consider the case of random look direction mismatch. We assume that the random look direction mismatch of the DS is distributed in $\left[-4^{\circ}, 4^{\circ}\right]$. In such case, the actual DS direction is randomly produced from $\left[1^{\circ}, 9^{\circ}\right]$. It should be noted that the random look direction mismatch changes from run to run while keeping constant from snapshot to snapshot. Figures 4 and 5 display the output SINR versus the SNR with fixed snapshots

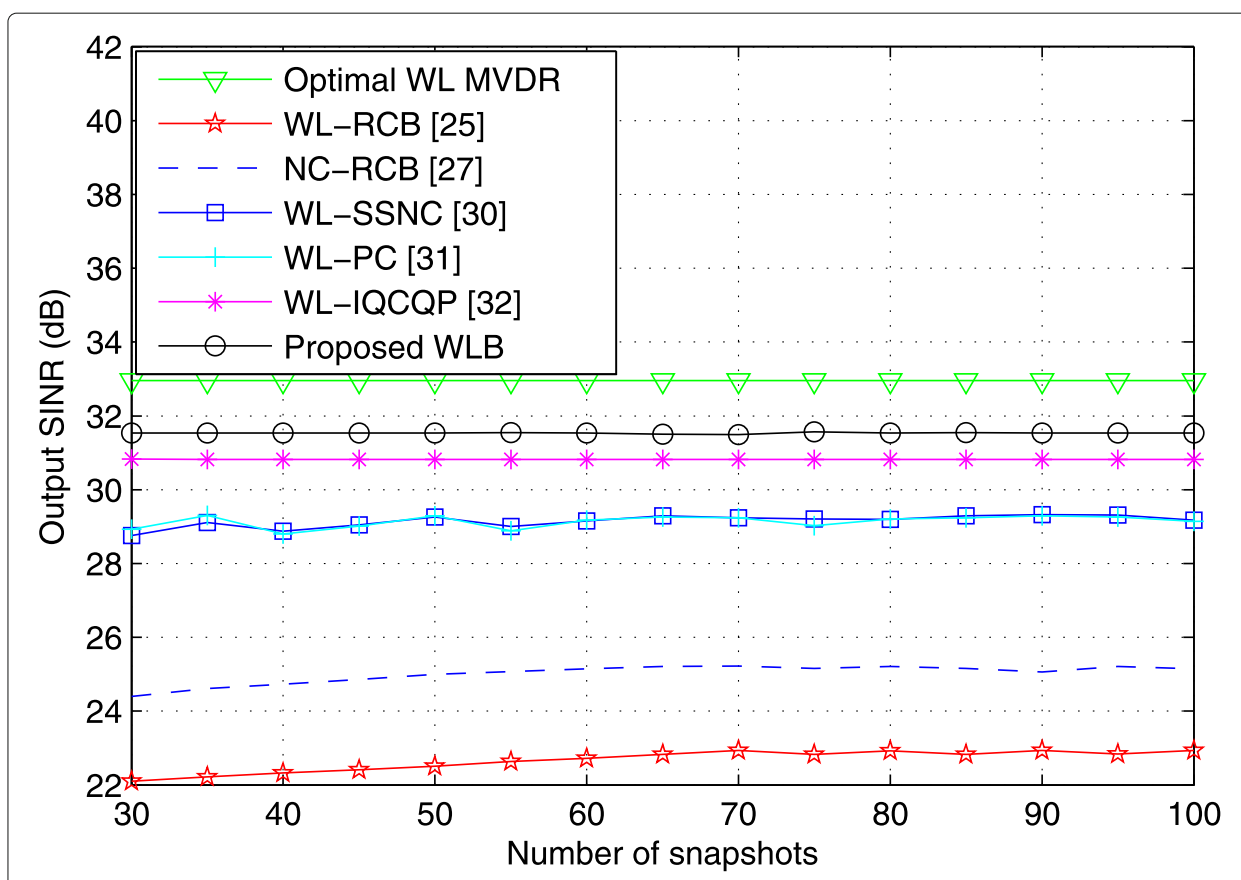

Fig. 5 Example 2. Output SINR of all WLBs versus the number of snapshots $K$ 
number $K=100$ and versus the number of snapshots with fixed $S N R=20 \mathrm{~dB}$, respectively. It can be seen that the proposed WLB yields higher SINRs than that of the other beamformers, which demonstrates that the proposed WLB is robust against the random look direction mismatch. This is because the proposed WLB not only reconstructs precise EINCM but also estimates accurate EDSSV. The WL-RCB and the NC-RCB have performance degradation owing to the exploitation of extended SCM and the steering vector mismatch caused by random look direction mismatch.

Example 3 We simulate the scenario where the mismatch is caused by the coherent local scattering. Under this type of mismatch, the actual DSSV is given by

$$
\mathbf{a}_{1}=\mathbf{a}\left(\bar{\theta}_{1}\right)+\sum_{p=1}^{4} e^{j \varphi_{p}} \mathbf{d}\left(\theta_{p}\right),
$$

where $\mathbf{a}\left(\bar{\theta}_{1}\right)$ is the direct path with assumed direction $\bar{\theta}_{1}$ and $\mathbf{d}\left(\theta_{p}\right)(p=1,2,3,4)$ are the coherently scattered paths. $\varphi_{p}(p=1,2,3,4)$ are the scattered path phases that are randomly produced from $[0,2 \pi] . \theta_{p}(p=1,2,3,4)$ are the scattered path directions that are independently produced from a random generator $\mathcal{N}\left(3^{\circ}, 1^{\circ}\right)$. Note that $\varphi_{p}$ and $\theta_{p}, p=1,2,3,4$ change in each simulation run but all of them remain fixed over snapshots. The performance curves versus the input SNR with fixed snapshots number $K=100$ and versus the number of snapshots with fixed $S N R=20 \mathrm{~dB}$ are depicted in Figs. 6 and 7 , respectively. Compared with the optimal WL MVDR, the proposed WLB encounters some performance loss resulted from the influence of coherent local scattering. Nevertheless, the performance of the proposed WLB is still superior to the other WLBs because of the precise EINCM reconstruction and the accurate EDSSV estimation.

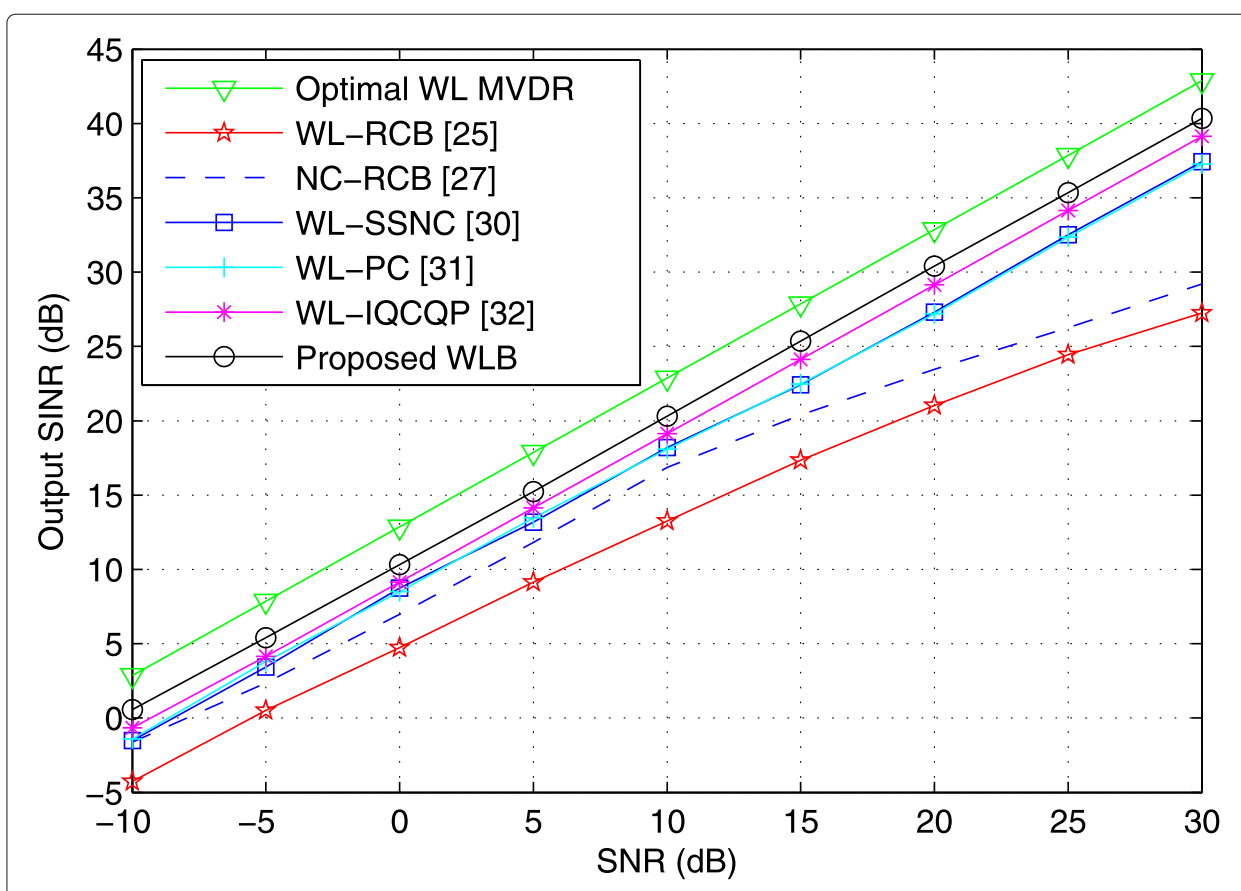

Fig. 6 Example 3. Output SINR of all WLBs versus the input SNR 


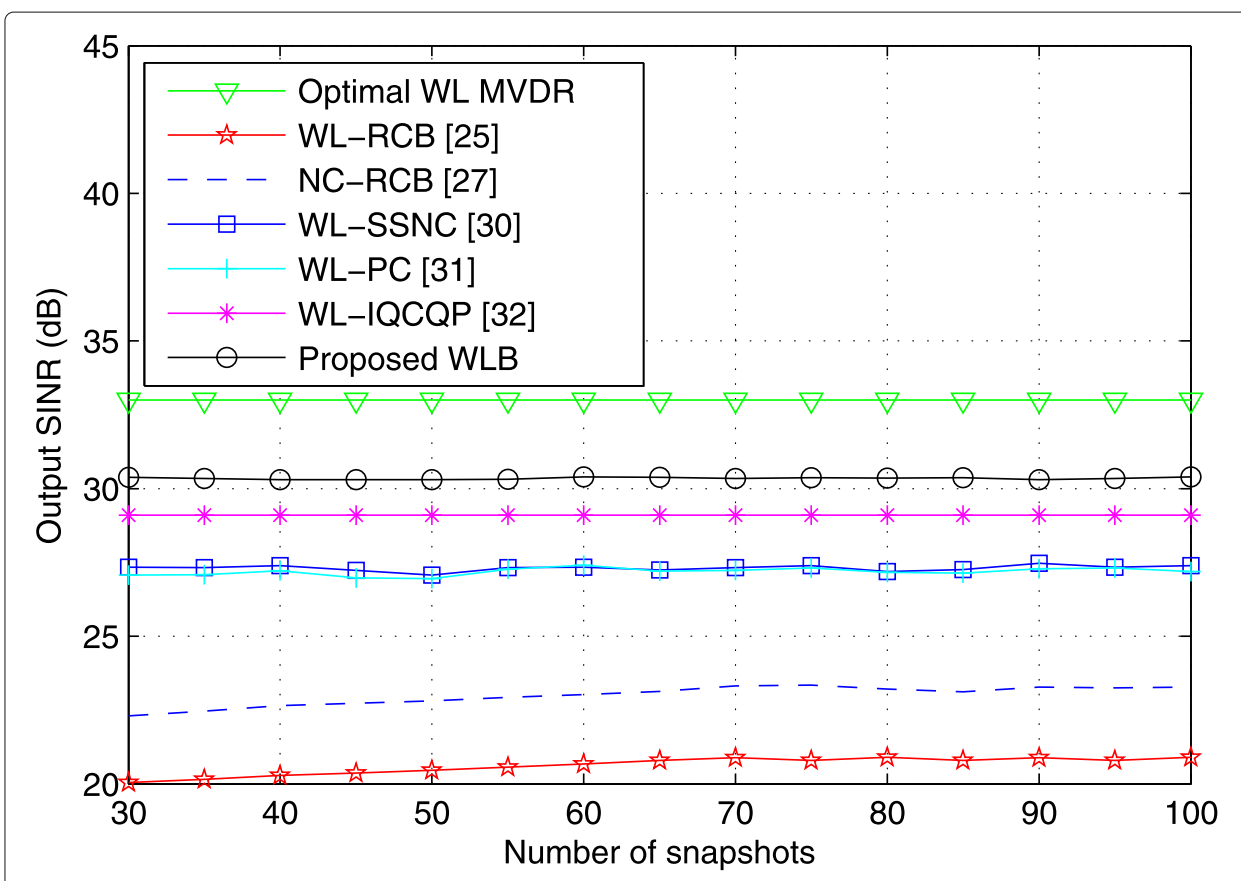

Fig. 7 Example 3. Output SINR of all WLBs versus the number of snapshots $K$

Example 4 We evaluate the beamforming performance in the presence of random steering vector mismatch. The actual DSSV is assumed to be randomly distributed in an uncertainty set, which is expressed by

$$
\mathbf{a}_{1}=\mathbf{a}\left(\bar{\theta}_{1}\right)+\frac{\xi}{\sqrt{N}}\left[e^{j \varphi_{1}}, e^{j \varphi_{2}}, \cdots, e^{j \varphi_{N}}\right]^{T},
$$

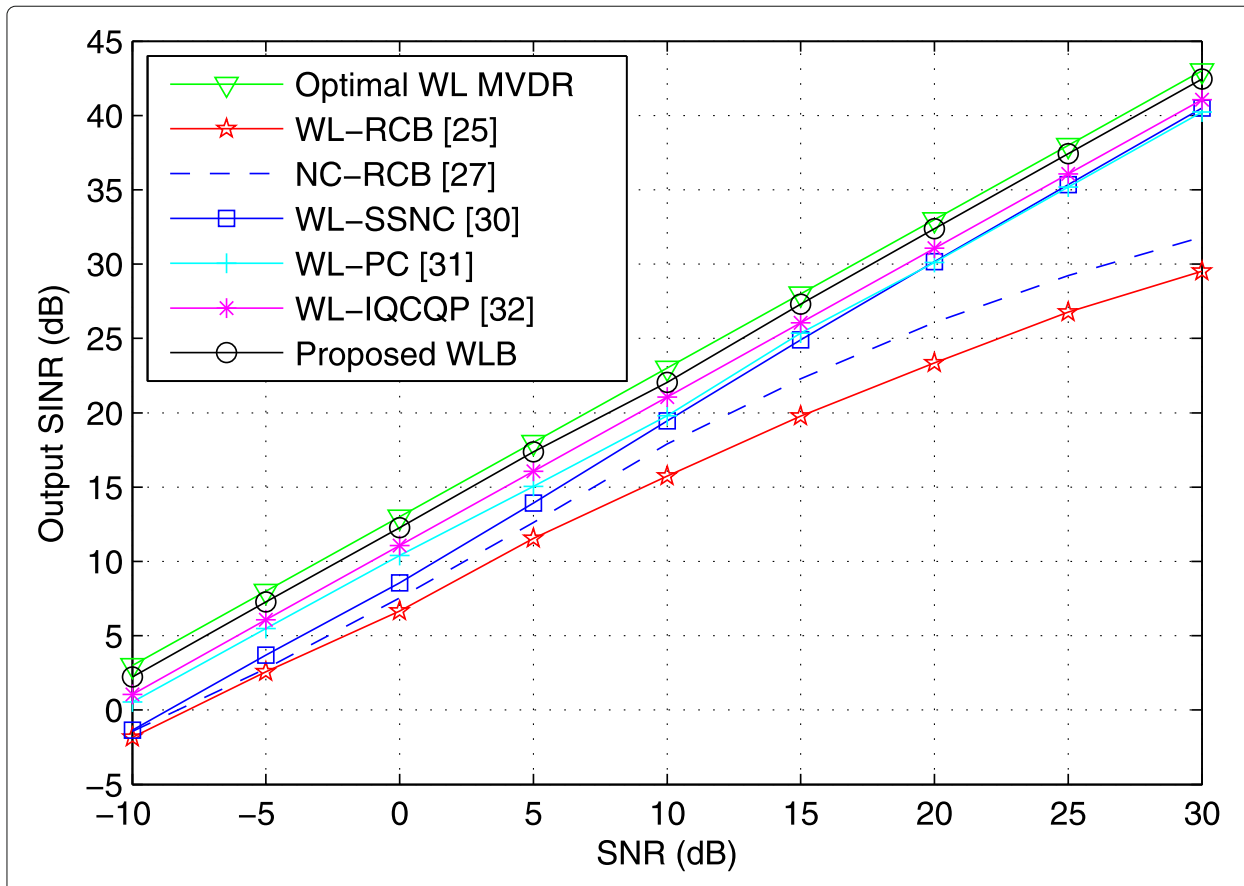

Fig. 8 Example 4. Output SINR of all WLBs versus the input SNR 
where $\mathbf{a}\left(\bar{\theta}_{1}\right)$ denotes the presumed DSSV and $\xi$ denotes the norm of random steering vector mismatch which is randomly produced from the interval $[0, \sqrt{0.5}]$ in each simulation run. $\varphi_{p}(p=1, \cdots, N)$ are the phases of the pth coordinate that are independently generated from the interval $[0,2 \pi]$ in each simulation run. Figures 8 and 9 plot the output SINR curves versus the input SNR with fixed snapshots number $K=100$ and versus the number of snapshots with fixed $S N R=20 \mathrm{~dB}$, respectively. We can observe that the output SINRs of the proposed WLB are close to that of the optimal WL MVDR in the whole range of input SNR and snapshots number, which means that the proposed WLB is effective for the noncircular signals. In addition, the proposed WLB performs better than the other WLBs, which illustrates that the proposed WLB can deal with the random steering vector mismatch.

Example 5 We take the mismatch caused by the wavefront distortion into consideration. We assume that the independent-increment phase distortions are accumulated by the entries of DSSV. The phase increments remain fixed in each simulation run that are independently produced from a random generator $\mathcal{N}(0,0.04)$. The output SINR curves versus the input SNR with fixed snapshots number $K=100$ and versus the number of snapshots with fixed $S N R=20 \mathrm{~dB}$ are plotted in Figs. 10 and 11, respectively. It can be found that the proposed WLB almost achieves the optimal beamforming performance, which demonstrates that the reconstruction of EINCM is precise and the estimation of EDSSV is accurate. In addition, the proposed WLB enjoys the best beamforming performance, which means that the proposed WLB can provide robustness against the wavefront distortion mismatch.

\section{Conclusion}

We proposed a robust WLB by combining the precise reconstruction of EINCM and the low-complexity estimation of EDSSV. By estimating the steering vectors, powers and

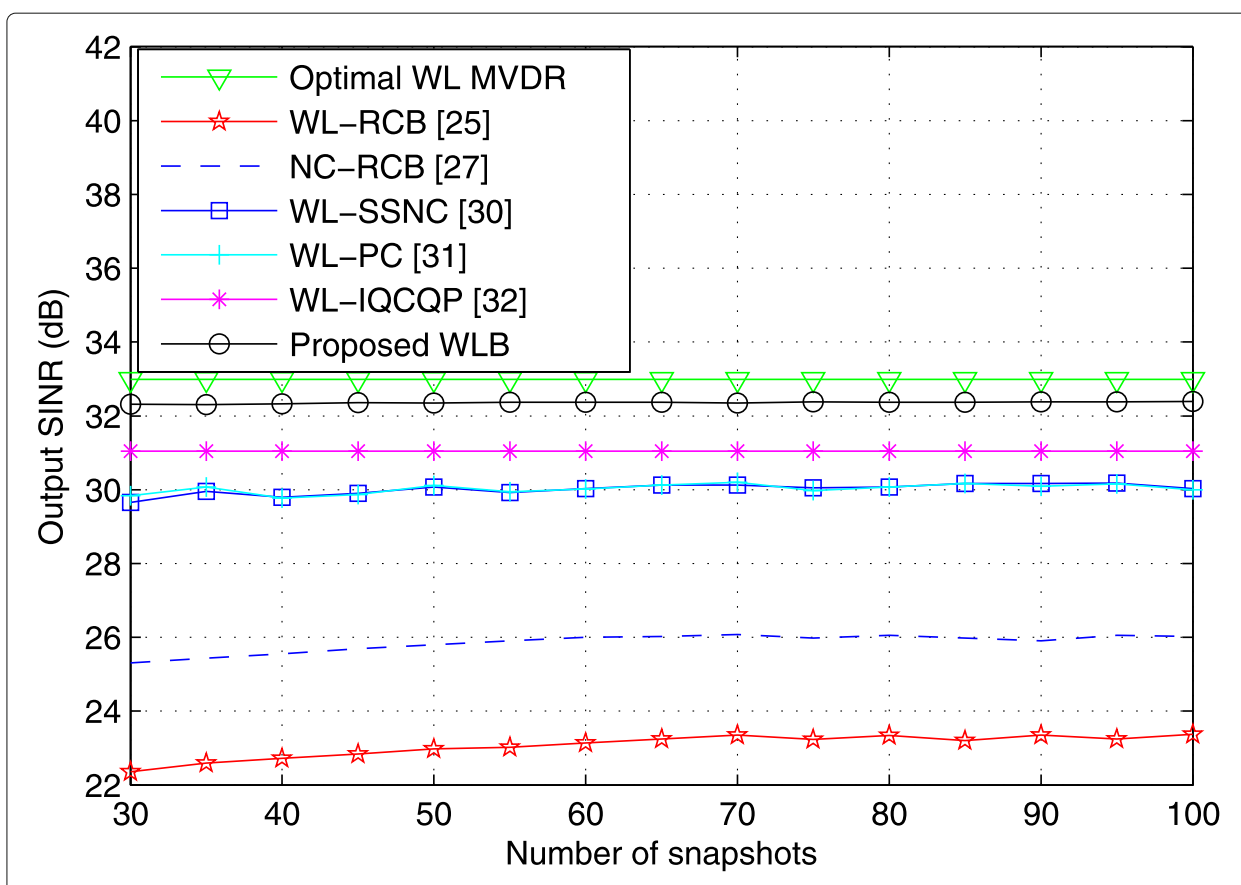

Fig. 9 Example 4. Output SINR of all WLBs versus the number of snapshots $K$ 


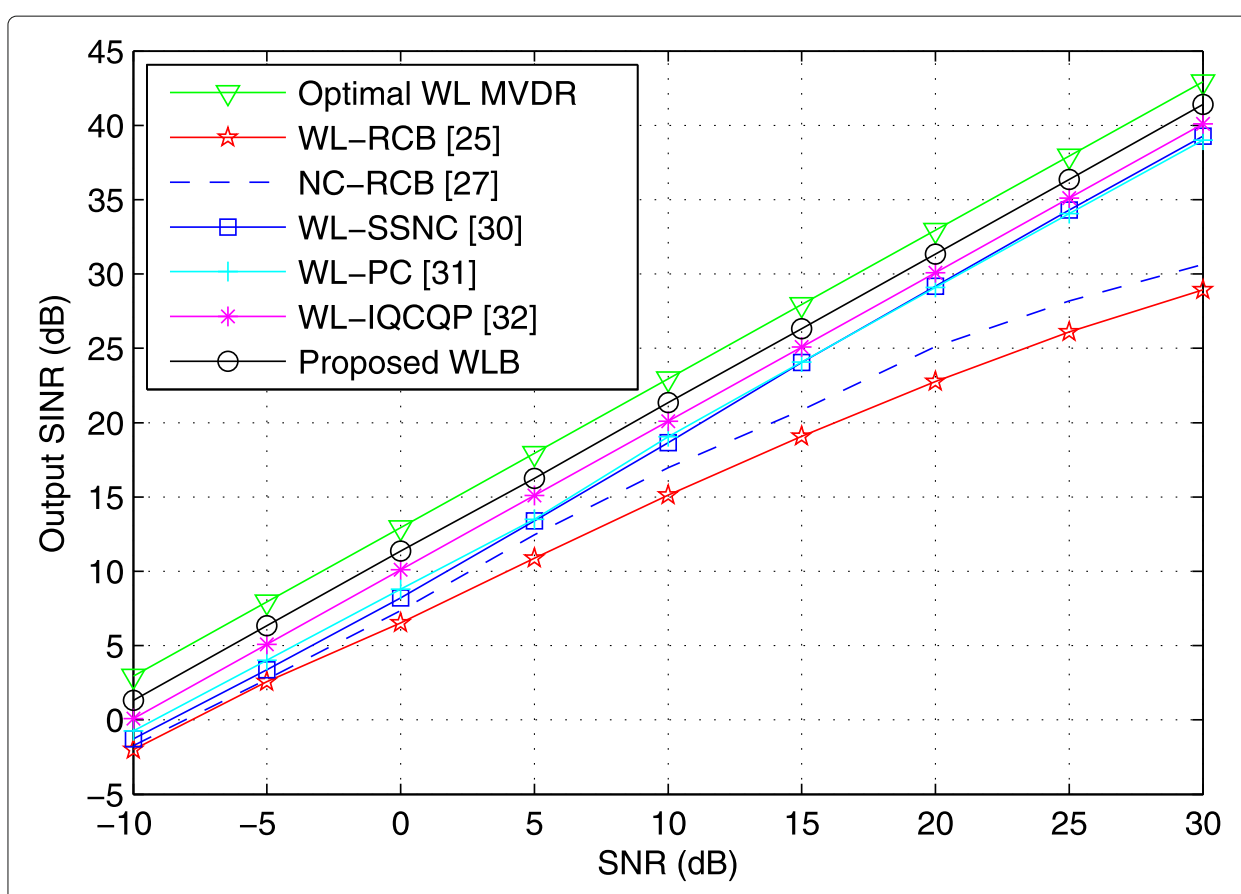

Fig. 10 Example 5. Output SINR of all WLBs versus the input SNR

noncircularity coefficients of all signals and the noise power, we propose to reconstruct the INCM and the pseudo INCM accurately using their definitions. These accurate estimates allow us to reconstruct the EINCM precisely. Moreover, we proposed to estimate the EDSSV from the intersection of two extended subspaces which has a closed-from expression and requires lower computational complexity than the traditional convex

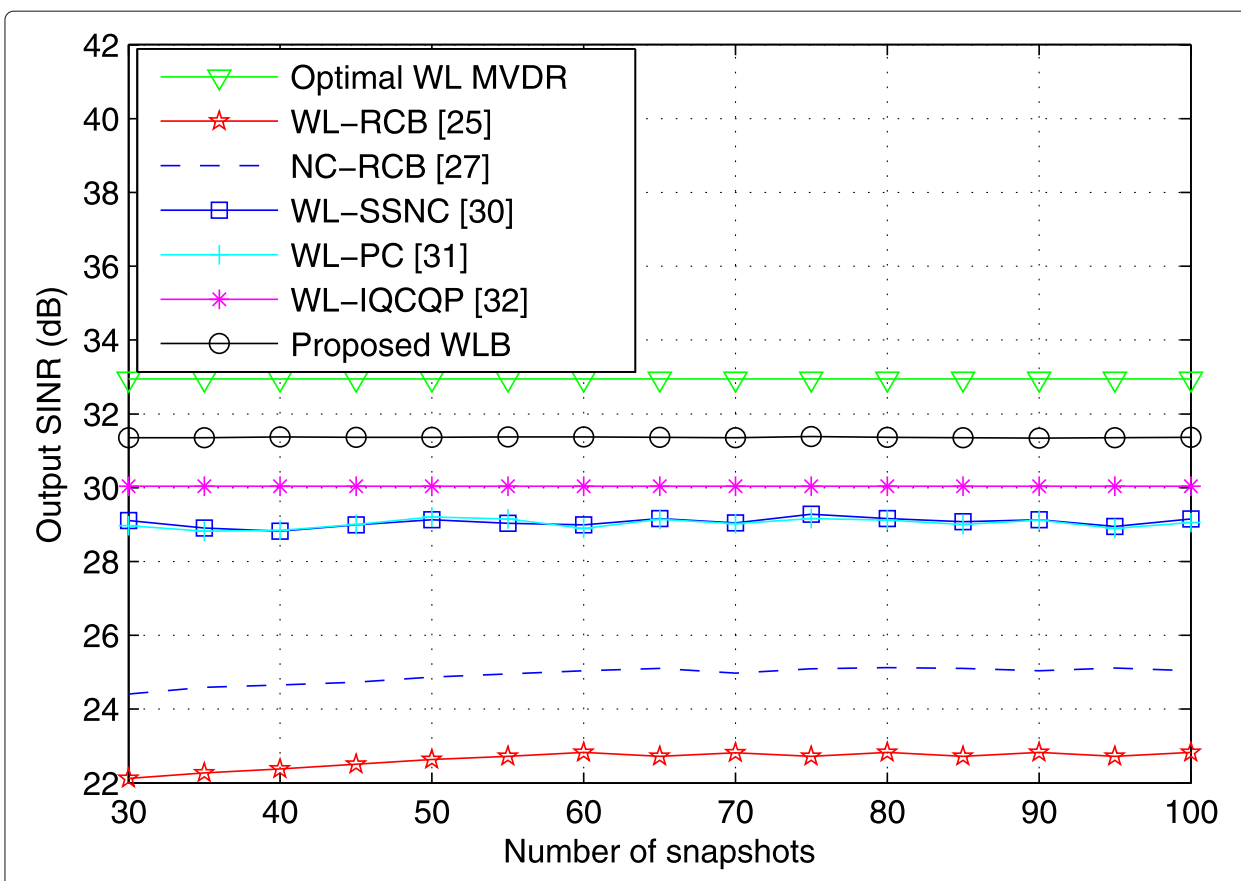

Fig. 11 Example 5. Output SINR of all WLBs versus the number of snapshots $K$ 
optimization methods. Simulation results demonstrate that the proposed WLB can provide robust performance against fixed look direction, random look direction, coherent local scattering, random steering vector, and wavefront distortion mismatches.

\begin{abstract}
Abbreviations
WLB: Widely linear beamformer; EINCM: Extended interference-plus-noise covariance matrix; INCM: Interference-plus-noise covariance matrix; EDSSV: Extended desired signal steering vector; DS: Desired signal; MVDR: Minimum variance distortionless response; LCMV: Linearly constrained minimum variance; UQPSK: Unbalanced quaternary phase shift keying; AM: Amplitude modulated; ASK: Amplitude-shift keying; BPSK: Binary phase-shift keying; WL: Widely linear; SNR: Signal-to-noise ratio; DSSV: Desired signal steering vector; SCM: Sample covariance matrix; SSNC: Spatial spectrum of noncircularity coefficient; PC: Projection constraint; SINR: Signal-to-interference-plus-noise ratio; IRCB: Iterative robust Capon beamformer; ULA: Uniform linear array; INR: Interference-to-noise ratio; NC-RCB: Noncircular robust Capon beamformer; IQCQP: Iterative quadratically constrained quadratic programming
\end{abstract}

\title{
Acknowledgements
}

This work was supported by the China Scholarship Council under Grant (No. 201806680057).

\section{Authors' contributions}

All authors have contributed to this paper. All authors read and approved the final manuscript.

\section{Funding}

This work was supported by the China Scholarship Council under Grant (No. 201806680057).

\section{Availability of data and materials}

The datasets generated and analyzed are available from the corresponding author on reasonable request.

\section{Competing interests}

The authors declare that they have no competing interests.

\section{Author details}

${ }^{1}$ College of Automation, Harbin Engineering University, 150001 Harbin, People's Republic of China. ${ }^{2}$ Department of Electrical and Computer Engineering, Queen's University, K7L 3N6 Kingston, Canada.

Received: 17 February 2020 Accepted: 2 October 2020

Published online: 17 October 2020

\section{References}

1. Y. Huang, S. A. Vorobyov, Z. Q. Luo, Quadratic matrix inequality approach to robust adaptive beamforming for general-rank signal model. IEEE Trans. Signal Process. 68, 2244-2255 (2020)

2. Z. Zheng, T. Yang, W. Q. Wang, H. C. So, Robust adaptive beamforming via simplified interference power estimation. IEEE Trans. Aerosp. Electron. Syst. 55(6), 3139-3152 (2019)

3. X. Zhang, Z. He, B. Liao, X. Zhang, W. Peng, Robust quasi-adaptive beamforming against direction-of-arrival mismatch. IEEE Trans. Aerosp. Electron. Syst. 54(3), 1197-1207 (2017)

4. Z. Meng, F. Shen, W. Zhou, Robust adaptive beamforming using the cyclostationarity of the source signals. Electron. Lett. 53(13), 858-860 (2017)

5. Z. Meng, F. Shen, W. Zhou, W. Lu, Robust adaptive beamforming based on subspace method. Electromagn, J., Waves Appl. 32(11), 1369-1378 (2018)

6. L. Yang, M. R. McKay, R. Couillet, High-dimensional MVDR beamforming: optimized solutions based on spiked random matrix models. IEEE Trans. Signal Process. 66(7), 1933-1947 (2018)

7. Z. Meng, F. Shen, W. Zhou, Iterative adaptive approach to interference covariance matrix reconstruction for robust adaptive beamforming, IET Microw. Antennas Propag. 12(10), 1704-1708 (2018)

8. Z. Meng, W. Zhou, Robust adaptive beamforming using iterative adaptive approach. J. Electromagn. Waves Appl. 33(4), 504-519 (2019)

9. B. Liao, C. Guo, L. Huang, Q. Li, H. C. So, Robust adaptive beamforming with precise main beam control. IEEE Trans. Aerosp. Electron. Syst. 53(1), 345-356 (2017)

10. S. Claessens, N. Pan, M. Rajabi, D. Schreurs, S. Pollin, Enhanced biased ASK modulation performance for SWIPT with AWGN channel and dual-purpose hardware. IEEE Trans. Microw. Theory Techn. 66(7), 3478-3486 (2018)

11. D. Zhu, V. J. Mathews, D. H. Detienne, A likelihood-based algorithm for blind identification of QAM and PSK signals. IEEE Trans. Wirel. Commun. 17(5), 3417-3430 (2018)

12. L. N. Ribeiro, A. L. F. de Almeida, J. C. M. Mota, Separable linearly constrained minimum variance beamformers. Signal Process. 158, 15-25 (2019)

13. G. Hu, L. Xu, P. Zhao, W. Wang, Confidence evaluation of BPSK signal analysis based on EVT. IEEE Trans. Aerosp. Electron. Syst. 56(2), 1515-1537 (2019)

14. H. Qian, K. Liu, W. Wang, Shrinkage widely linear recursive least square algorithms for beamforming. IEICE Trans. Commun. 99(7), 1532-1540 (2016)

15. A. Song, A. Wang, S. Luan, T. Qiu, Widely linear generalized sidelobe canceling beamforming with variable diagonal loading. AEU Int. J. Electron. Commun. 76, 77-85 (2017)

16. X. Wu, Y. Cai, M. Zhao, R. C. de Lamare, B. Champagne, Adaptive widely linear constrained constant modulus reduced-rank beamforming. IEEE Trans. Aerosp. Electron. Syst. 53(1), 477-492 (2017) 
17. Y. Xia, D. P. Mandic, Augmented performance bounds on strictly linear and widely linear estimators with complex data. IEEE Trans. Signal Process. 66(2), 507-514 (2017)

18. A. E. Stott, S. Kanna, D. P. Mandic, Widely linear complex partial least squares for latent subspace regression. Signal Process. 152, 350-362 (2018)

19. S. Zhang, J. Zhang, W. X. Zheng, H. C. So, Widely linear complex-valued estimated-input LMS algorithm for bias-compensated adaptive filtering with noisy measurements. IEEE Trans. Signal Process. 67, 3592-3605 (2019)

20. T. Nitta, M. Kobayashi, D. P. Mandic, Hypercomplex widely linear estimation through the lens of underpinning geometry. IEEE Trans. Signal Process. 67(15), 3985-3994 (2019)

21. P. Chevalier, A. Blin, Widely linear MVDR beamformers for the reception of an unknown signal corrupted by noncircular interference. IEEE Trans. Signal Process. 55(11), 5323-5336 (2007)

22. P. Chevalier, J. P. Delmas, A. Oukaci, in 2009 IEEE International Conference on Acoustics, Speech and Signal Processing, Optimal widely linear MVDR beamforming for noncircular signals (IEEE, Taipei, 2009), pp. 3573-3576

23. P. Chevalier, J. P. Delmas, A. Oukaci, Properties, performance and practical interest of the widely linear MMSE beamformer for nonrectilinear signals. Signal Process. 97(7), 269-281 (2014)

24. Y. M. Shi, L. Huang, C. Qian, H. C. So, Shrinkage linear and widely linear complex-valued least mean squares algorithms for adaptive beamforming. IEEE Trans. Signal Process. 63(1), 119-131 (2015)

25. G. Wang, J. P. Lie, M. C.See S., in 2012 IEEE International Conference on Acoustics, Speech and Signal Processing, A robust approach to optimum widely linear MVDR beamformer (IEEE, Kyoto, 2012), pp. 2593-2596

26. D. Xu, L. Huang, X. Xu, Z. Ye, Widely linear MVDR beamformers for noncircular signals based on time-averaged second-order noncircularity coefficient estimation. IEEE Trans. Veh. Technol. 62(7), 3219-3227 (2014)

27. F. Wen, Q. Wan, H. Wei, R. Fan, Y. Luo, Robust Capon beamforming exploiting the second-order noncircularity of signals. Signal Process. 102(102), 100-111 (2014)

28. L. Huang, J. Zhang, L. Zhang, Z. Ye, Widely linear minimum dispersion beamforming for sub-Gaussian noncircular signals. Signal Process. 122, 123-128 (2015)

29. Y. Xu, Y. Huang, J. Liu, Z. Liu, Non-circularity coefficient estimation of the SOI for narrowband widely linear beamforming. IET Microw. Antennas Propag. 13(5), 649-659 (2019)

30. D. Xu, C. Gong, S. Cao, X. Xu, Z. Ye, Robust widely linear beamforming based on spatial spectrum of noncircularity coefficient. Signal Process. 104(6), 167-173 (2014)

31. J. Zhang, L. Huang, L. Zhang, B. Zhang, in 2015 IEEE International Conference on Acoustics, Speech and Signal Processing, Robust widely linear beamformer based on a projection constraint (IEEE, Brisbane, 2015), pp. 2509-2513

32. J. Liu, W. Xie, Q. Wan, G. Gui, Robust widely linear beamforming via the techniques of iterative QCQP and shrinkage for steering vector estimation. IEEE Access. 6, 3-17152 (2018)

33. Y. Gu, N. A. Goodman, S. Hong, Y. Li, Robust adaptive beamforming based on interference covariance matrix sparse reconstruction. Signal Process. 96(5), 375-381 (2014)

34. J.P. Lie, W. Ser, M. C.See S., Adaptive uncertainty based iterative robust Capon beamformer using steering vector mismatch estimation. IEEE Trans. Signal Process. 59(9), 4483-4488 (2011)

35. L. Chang, C. C. Yeh, Performance of DMI and eigenspace-based beamformers. IEEE Trans. Antennas Propag. 40(11), 1336-1347 (1992)

36. Y. Gu, A. Leshem, Robust adaptive beamforming based on interference covariance matrix reconstruction and steering vector estimation. IEEE Trans. Signal Process. 60(7), 3881-3884 (2012)

37. S. Mohammadzadeh, O. Kukrer, Robust adaptive beamforming with improved interferences suppression and a new steering vector estimation based on spatial power spectrum. Circuits Syst. Signal Process. 38(9), 4162-4179 (2019)

38. J. Zhuang, A. Manikas, Interference cancellation beamforming robust to pointing errors. IET Signal Process. 7(2), 120-127 (2013)

39. M. H. Er, A. Cantoni, A new set of linear constraints for broadband time-domain element-space processors. IEEE Trans. Antennas Propag. 34(3), 320-329 (1986)

40. J. Gu, H. Stark, Y. Yang, Wide-band smart antenna design using vector space projection methods. IEEE Trans. Antennas Propag. 52(12), 3228-3236 (2004)

41. F. Zhang, Q. Zhang, Eigenvalue inequalities for matrix product. IEEE Trans. Autom. Control. 51(9), 1506-1509 (2006)

\section{Publisher's Note}

Springer Nature remains neutral with regard to jurisdictional claims in published maps and institutional affiliations.

\section{Submit your manuscript to a SpringerOpen ${ }^{\circ}$ journal and benefit from:}

- Convenient online submission

Rigorous peer review

- Open access: articles freely available online

- High visibility within the field

- Retaining the copyright to your article

Submit your next manuscript at $\gg$ springeropen.com 